\title{
Application of triple compound combination anti-synchronization among parallel fractional snap systems \& electronic circuit implementation
}

\author{
Emad E. Mahmoud ${ }^{1,2^{*}}$ (D), Pushali Trikha ${ }^{3}$, Lone Seth Jahanzaib ${ }^{3}$, M. Higazy ${ }^{1,4}$ and Monagi H. Alkinani ${ }^{5}$
}

\author{
"Correspondence: \\ e.mahmoud@tu.edu.sa; \\ emad_eluan@yahoo.com \\ 'Department of Mathematics and \\ Statistics, College of Science, Taif \\ University, P.O. Box 11099, Taif \\ 21944, Saudi Arabia \\ ${ }^{2}$ Department of Mathematics, \\ Faculty of Science, Sohag University, \\ Sohag 82524, Egypt \\ Full list of author information is \\ available at the end of the article
}

\begin{abstract}
In this article we examine the dynamical properties of the fractional version of the snap system by means of chaotic attractor, existence, and uniqueness of the solution, symmetry, dissipativity, stagnation point analysis, Lyapunov dynamics, K.Y. dimension, bifurcation diagram, etc. Also, parallel systems to this system are synchronized in presence of uncertainties and external disturbances using triple compound combination anti-synchronization by two ways. Synchronization time is compared with some other works. Also the utilization of achieved synchronization is illustrated in secure transmission. By constructing the snap system's signal flow graph and its real electronic circuit, some of its additional invariants are investigated.
\end{abstract}

Keywords: Dynamical properties; Electronic circuit; Parallel systems; Triple compound combination anti-synchronization

\section{Introduction}

Chaos is the common attribute of nonlinear dynamical systems. It has some interesting features such as irregular, complex, and unpredictable behavior. It was in 1960 while studying atmospheric turbulence that Lorenz $[25,26]$ realized that the 3-D differential equation system possessed a very irregular chaos like solution. After the Lorenz system, the Lu system and Chen system were proposed which too had complex dynamical behavior. In order to utilize chaos, many methods have been developed by researchers, such as chaos control, suppression, and synchronization, which led to the revolution in chaos theory. Because of these developments, chaotic systems found many applications across many disciplines such as secure communication, robotics, weather systems, encryption, neurons, circuits, oscillators, ecology, biology, and finance systems. Chaos synchronization, in which two or more chaotic systems are made to follow the same path on application of suitable controllers, was introduced in 1990. Synchronization of chaos involves synchronizing systems which are highly sensitive to initial conditions (I.C.) and parametric values. Many synchronization techniques [17, 19-22, 27, 30,31, 35, 54] have been developed since then using various control methods. Table 1 gives an idea of how new synchronization

(c) The Author(s) 2021. This article is licensed under a Creative Commons Attribution 4.0 International License, which permits use, sharing, adaptation, distribution and reproduction in any medium or format, as long as you give appropriate credit to the original author(s) and the source, provide a link to the Creative Commons licence, and indicate if changes were made. The images or other third party material in this article are included in the article's Creative Commons licence, unless indicated otherwise in a credit line to the material. If material is not included in the article's Creative Commons licence and your intended use is not permitted by statutory regulation or exceeds the permitted use, you will need to obtain permission directly from the copyright holder. To view a copy of this licence, visit http://creativecommons.org/licenses/by/4.0/. 
Table 1 Different Types of Synchronization Techniques

\begin{tabular}{llr}
\hline Type of synchronization & Authors & Year \\
\hline Complete [36] & Pecora, Carroll & 1990 \\
Generalized [42] & Rulkov, Sushchik,Tsimring, Abarbanel & 1995 \\
Phase [40] & Rosenblum, Pikovsky, Kurths & 1996 \\
Lag [41] & Rosenblum, Pikovsky, Kurths & 1997 \\
Projective [32] & Mainieri, Rehacek & 1999 \\
Intermittent lag [3] & Boccaletti \& Valladares & 2000 \\
Combination [43] & Runzi, Yinglan, Shucheng & 2011 \\
Compound [50] & Sun, Shen, Yin \& Xu & 2013 \\
Difference [10] & Dongmo, Ojo, Woafo, Njah & 2018 \\
\hline
\end{tabular}

techniques have been developing every now and then. Many new chaotic dynamical systems $[37,56,58]$ of finite dimension are being developed in chaos theory. Chaotic systems are also studied on the basis of attractors, hidden attractors, multi-scroll attractors, selfexcited attractors, double-wing, etc. Designing of chaotic system with some special characteristics has become an attractive topic of research in recent years. For example, chaotic systems having only one stable equilibrium, surfaces or curves or planes of equilibria, no equilibria, non-hyperbolic equilibria, etc. As the fractional order adds an extra dimension in dynamics of chaotic systems, a fractional version of Lu system, Liu system, Rossler system etc. is being studied. Nowadays fractional discrete time chaotic systems [61-64] are also being studied. Fractional calculus has been used recently to study nonlinear fractional B.V.P. [16], biological snap oscillator [44], harmonic oscillator, etc. [1, 2, 15, 34].

In chaos applications, a very important topic is the hardware realization of mathematical models [5].

The graph $H(V, E)$ is defined as a pair of two sets: vertices set $V$ and edges set $E$ and written as $H$ for simplicity. The eigenvalues (E.V.) of the adjacency matrix of $H$ are said to be the E.V. of $H$ and form its spectrum. A graph of order $n=|V|$ has $n$ eigenvalues; we denote these by $\lambda_{1}, \ldots, \lambda_{n}$. The basics of graph spectrum can be found in [45]. The graph spectrum can be used to calculate a very useful graph invariant, namely the graph energy $E(H)[7,8]$ where

$$
E(H)=\sum_{i=1}^{n}\left|\lambda_{i}\right|
$$

Motivated by the above discussion, here the fractional version of the snap system with only one nonlinear term is dynamically analyzed using tools like Lyapunov exponents (L.E.) [4, 13, 59], Kaplan York (K.Y.) dimension, bifurcations, etc. The system is synchronized with its parallel systems considering uncertainties and external disturbances in triple compound combination anti-synchronization. The synchronization method is illustrated in secure communication $[6,12]$ by an example. Synchronization time is compared with some other works. Also the electronic circuit of the snap system is realized to assure its real life applications.

Summarizing the paper:

1. A fractional version of the previously studied integer order dimensional chaotic system is studied. The system is explored using stagnation points analysis, symmetric character, Lyapunov dynamics, bifurcation analysis, etc.

2. The effect of fractional order on system dynamics for varying fractional order in the 0.8 to 1 range is studied. 
3. A novel technique-triple compound combination anti-synchronization-is developed to anti-synchronize two leader systems and six follower systems by two methods.

4. Bounds of uncertainties and disturbances are also estimated using the adaptive SMC technique.

5. Achieved results are compared with some earlier published literature.

6. Application of the achieved synchronization on the introduced fractional version of the snap system in secure transmission is illustrated.

7. Electronic circuit of the introduced snap system is also provided.

\section{The fractional snap system and its dynamics}

The integer order snap chaotic system was proposed in 2019 by Pham et al. [38] with only one quadratic nonlinear term given as follows:

$$
\begin{aligned}
& \dot{z_{1}}=z_{2}, \\
& \dot{z_{2}}=z_{3}, \\
& \dot{z_{3}}=z_{4}, \\
& \dot{z_{4}}=-a z_{1}-z_{2}-z_{4}+b z_{1} z_{3},
\end{aligned}
$$

where $z=\left(z_{1}, z_{2}, z_{3}, z_{4}\right)^{T} \in R^{4}$ are state variables.

Though many snap systems have been proposed by researchers over the time, developing new snap systems has always been of interest to researchers owing to their application in random number generations [11], cryptosystems [60], steganography [23], etc. Moreover, studying the fractional version of the snap system holds importance because fractional ordered systems have better memory properties and hence model real life situations more accurately than the integer counterparts. Also it is interesting to see their chaotic behavior vary for different fractional orders.

Among the many definitions of fractional derivatives, the following Caputo's definition is used:

$$
{ }_{t_{0}} D_{t}^{\alpha} f(t)=\frac{1}{\Gamma(n-\alpha)} \int_{t_{0}}^{t} \frac{f^{(n)}(\tau)}{(t-\tau)^{\alpha-n+1}} d \tau, \quad t>t_{0} .
$$

We here examine the fractional version of this newly introduced system:

$$
\begin{aligned}
& D^{q} z_{1}=z_{2}, \\
& D^{q} z_{2}=z_{3}, \\
& D^{q} z_{3}=z_{4}, \\
& D^{q} z_{4}=-a z_{1}-z_{2}-z_{4}+b z_{1} z_{3} .
\end{aligned}
$$

Figures 1 and 2 show the chaotic attractors and state trajectories for $a=0.2, b=1$ with I.C. $(0,0.1,0.1,0)$ and $q=0.95$.

Note: For numerical simulations we have used Grünwald-Letnikov approximation function in MATLAB software. 
Mahmoud et al. Advances in Difference Equations

(2021) 2021:211

Page 4 of 26

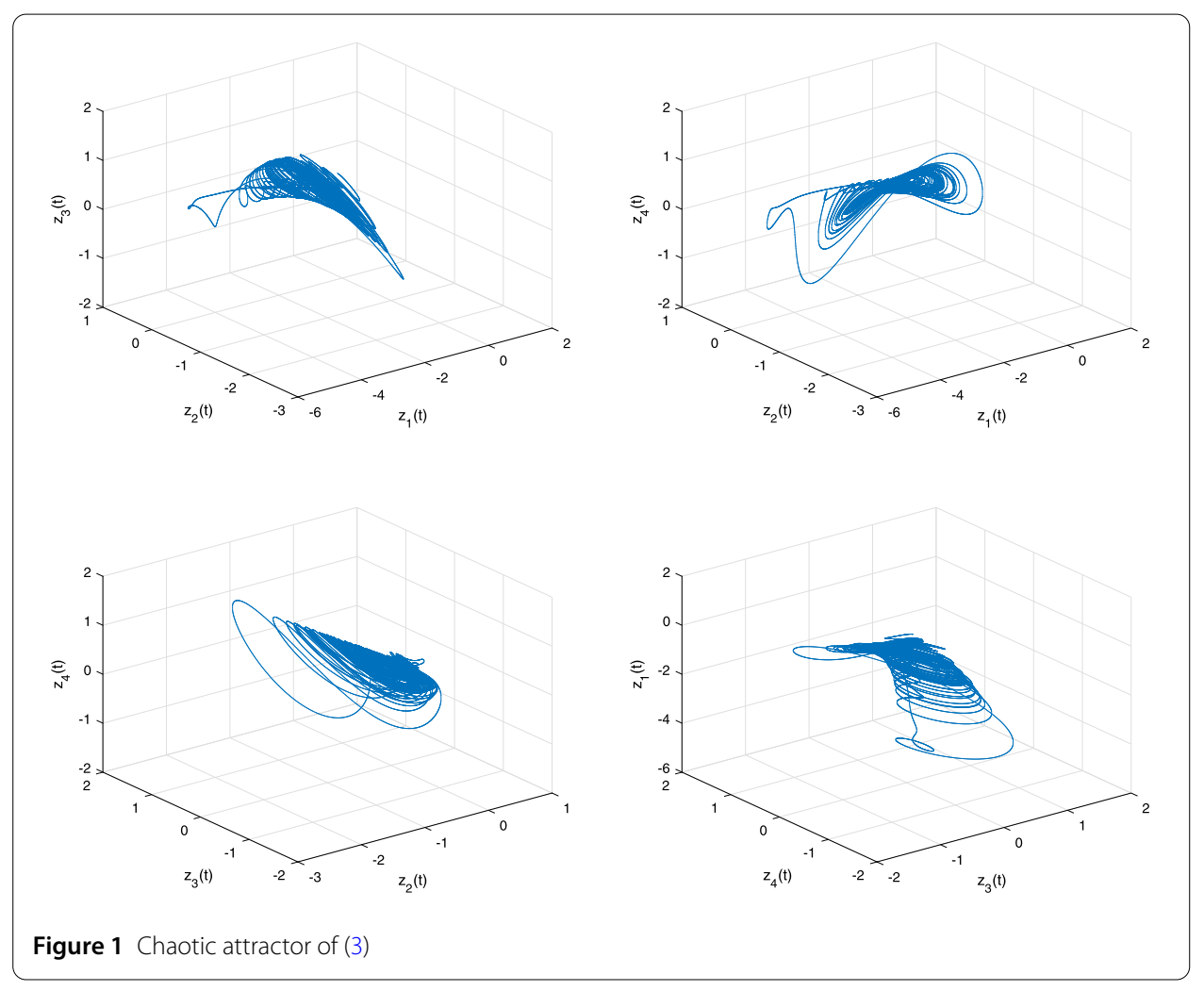

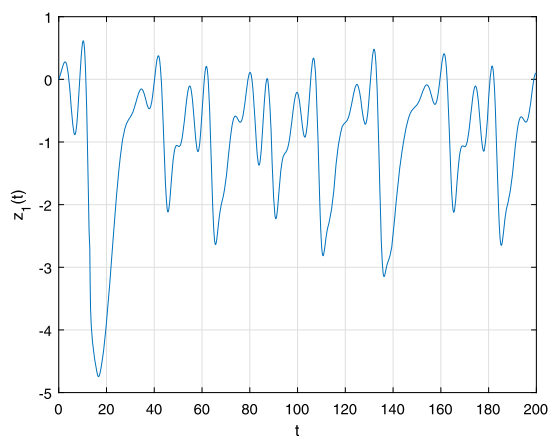

(a)

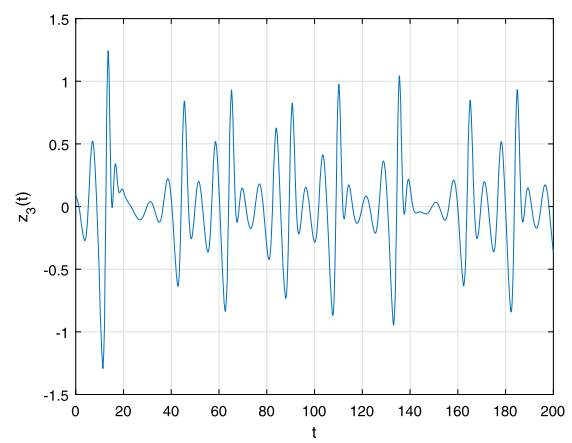

(c)

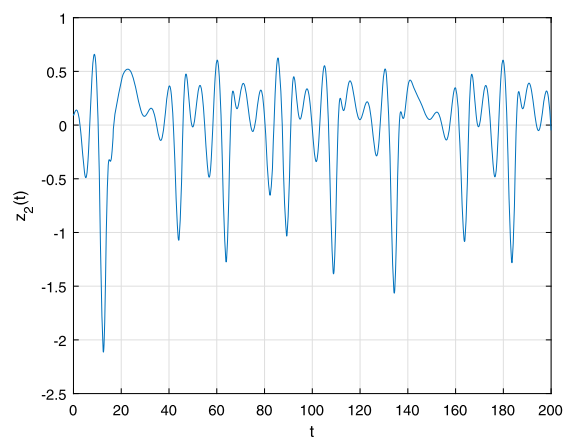

(b)

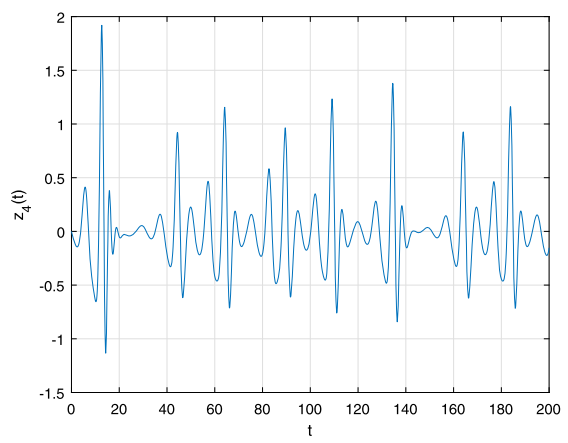

(d)

Figure 2 Trajectories of (3) 


\section{Solution of a novel system}

Theorem The I.V.P. of (3):

$$
\begin{aligned}
& D^{q} z(t)=B_{1} z(t)+z_{1}(t) B_{2} z(t), \\
& z(0)=z_{o}
\end{aligned}
$$

where $0<t \leq \tau, z(t)=\left(z_{1}(t), z_{2}(t), z_{3}(t), z_{4}(t)\right)^{T} \in \mathbb{R}^{4}, z_{o}=\left(z_{1 o}, z_{2 o}, z_{3 o}, z_{4 o}\right)^{T}, q=\left(q_{1}, q_{2}, q_{3}\right.$, $\left.q_{4}\right)^{T}, 0<q_{i}<1$, for $i=1,2,3,4$.

$$
B_{1}=\left[\begin{array}{cccc}
0 & 1 & 0 & 0 \\
0 & 0 & 1 & 0 \\
0 & 0 & 0 & 1 \\
-0.2 & -1 & 0 & -1
\end{array}\right] \quad \& \quad B_{2}=\left[\begin{array}{llll}
0 & 0 & 0 & 0 \\
0 & 0 & 0 & 0 \\
0 & 0 & 0 & 0 \\
0 & 0 & 1 & 0
\end{array}\right]
$$

for some $\tau>0$, then a unique solution is guaranteed.

Proof As $G(z)=B_{1} z+z_{1} B_{2} z$ is continuous on $\mathbb{R}^{4}$ and hence bounded on any compact interval $\left[z_{o}-\epsilon, z_{o}+\epsilon\right]$ for $\epsilon>0$, therefore the Lipschitz continuity on this interval is sufficient to apply the existence and uniqueness theorem in [9].

We have $|G(z)-G(w)|=\left|B_{1} z+z_{1} B_{2} z-B_{1} w-w_{1} B_{2} w\right|$.

As

$$
\left|z_{1}-w_{1}\right| \leq|z-w|,
$$

we get

$$
\begin{aligned}
\left|z_{1} B_{2} z-w_{1} B_{2} w\right| & =\left|B_{2}\left[z_{1}-w_{1}\right] z+B_{2}\left[w_{1}[z-w]\right]\right| \\
& \leq\left\|B_{2}\right\| \cdot\left[|z|+\left|w_{1}\right|\right](|z-w|),
\end{aligned}
$$

which implies that

$$
\begin{aligned}
|G(z)-G(w)| & \leq\left\|B_{1}\right\| \cdot|z-w|+\left\|B_{2}\right\|\left[2\left|z_{o}\right|+2 \epsilon\right](|z-w|) \\
& \leq\left(\left\|B_{1}\right\|+\left\|B_{2}\right\|\left[2\left|z_{o}\right|+2 \epsilon\right]\right)|z-w| \\
& \leq \psi|z-w|
\end{aligned}
$$

where $\psi=\left\|B_{1}\right\|+\left\|B_{2}\right\|\left[2\left|z_{o}\right|+2 \epsilon\right]$.

This implies that $\mathrm{G}(\mathrm{z})$ is Lipschitz continuous, and hence the system has a unique solution.

\section{Stagnation points, dissipativity, and symmetry}

We evaluate the stagnation points of (3) by equating $D^{q} z_{i}$ to zeroi.e.

$$
\begin{aligned}
& z_{2}=0, \\
& z_{3}=0,
\end{aligned}
$$




$$
\begin{aligned}
& z_{4}=0, \\
& -a z_{1}-z_{2}-z_{4}+b z_{1} z_{3}=0 .
\end{aligned}
$$

For $a=0.2, b=1$, we obtain a stagnation point $E=(0,0,0,0)$.

Theorem The stagnation point $E$ is an unstable saddle focus type stagnation point.

Proof Jacobian matrix $\mathbf{J}$ of (3) is

$$
\mathbf{J}=\left[\begin{array}{cccc}
0 & 1 & 0 & 0 \\
0 & 0 & 1 & 0 \\
0 & 0 & 0 & 1 \\
-0.2+z_{3} & -1 & b z_{1} & -1
\end{array}\right] .
$$

At $E, \mathbf{J}$ is

$$
\left[\begin{array}{cccc}
0 & 1 & 0 & 0 \\
0 & 0 & 1 & 0 \\
0 & 0 & 0 & 1 \\
-0.2 & -1 & 0 & -1
\end{array}\right]
$$

The eigenvalues of $\mathbf{J}$ at $E$ are

$$
\begin{aligned}
& \mu_{1}=-1.42393, \quad \mu_{2}=-0.194106, \quad \mu_{3}=0.309017+0.792537 i, \\
& \mu_{4}=0.309017-0.792537 i
\end{aligned}
$$

implying $E$ is an unstable saddle node type stagnation point.

Consider system (3) as

$$
\left[\begin{array}{c}
D^{q} z_{1} \\
D^{q} z_{2} \\
D^{q} z_{3} \\
D^{q} z_{4}
\end{array}\right]=\left[\begin{array}{c}
z_{2} \\
z_{3} \\
z_{4} \\
-a z_{1}-z_{2}-z_{4}+b z_{1} z_{3}
\end{array}\right] .
$$

We find the divergence of this vector field by

$$
\begin{aligned}
\nabla G & =\frac{\partial z_{2}}{\partial z_{1}}+\frac{\partial z_{3}}{\partial z_{2}}+\frac{\partial z_{4}}{\partial z_{3}}+\frac{\partial\left(-a z_{1}-z_{2}-z_{4}+b z_{1} z_{3}\right)}{\partial z_{4}} \\
& =-1
\end{aligned}
$$

i.e. $\nabla G<0$ implying that the system under consideration is dissipative for any values of the parameters.

Symmetric behavior about the $z_{3}$ axis is observed as the system remains invariant under the transformation $z_{1} \rightarrow-z_{1}, z_{2} \rightarrow-z_{2}, z_{3} \rightarrow z_{3}, z_{4} \rightarrow-z_{4}$. This implies rotational symmetry about the $z_{3}$ axis. However, the system does not remain invariant under such transformations about the $z_{1}, z_{2}, z_{4}$ axis respectively. 


\section{Lyapunov exponents}

L.E. is the rate at which trajectories which are very very close to each other diverge away. The positive L.E. confirms chaos. For $a=0.2, b=1$, and I.C. $(0,0.1,0.1,0)$, (3) has the following L.E.:

$$
0.107,-0.0002 \approx 0,-0.9791,-0.3707
$$

i.e. (3) is a chaotic dynamical system.

The chaotic attractor of (3) possesses a fractional dimension defined as follows:

$$
D_{Y K}=p+\frac{\sum_{j=1}^{p} L \cdot E_{\cdot j}}{\left|L \cdot E_{\cdot p+1}\right|+\left|L \cdot E_{\cdot p+2}\right|}
$$

Here, $p$ is the integer such that $\sum_{j=1}^{p} L . E_{. j} \geq 0$ and $\sum_{j=1}^{p+1} L . E_{. j}<0$.

The K.Y. dimension for system (3) is 2.079122 which is a noninteger value.

\section{Bifurcation analysis}

The system dynamics is significantly dependent on the parameters. Different parameters have different influence on equilibrium points and system's stability, hence on the dynamics of the system. Therefore observing the change in the system dynamics by varying a parameter in a range brings insight into the chaotic behavior over the specified range. The bifurcation diagram is obtained by plotting local maxima for changing parameter values in range.

For $a=0.2, b=1$. and I.C. $(0,0.1,0.1,0)$, Fig. 3(a) gives the bifurcation diagram for $a \in$ $(0,1)$ and Fig. 3(b) gives for $b \in(0.5,1.5)$. The increase in the density of points in Fig. 3(a) on crossing a particular value highlights its route to chaos, whereas the same density of points in Fig. 3(b) shows chaotic behavior throughout the range.

\section{System dynamics for variable fractional order}

In fractional order systems, the system shows much diverseness with respect to variable as shown in Fig. 5(a) and 5(b). The fractional order has significance in the system dynamics.

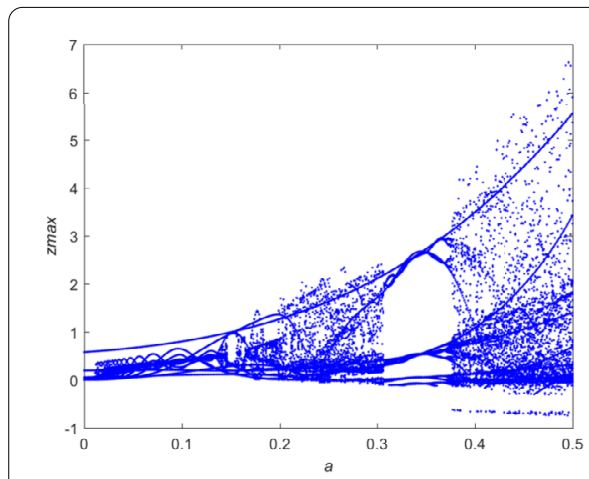

(a)

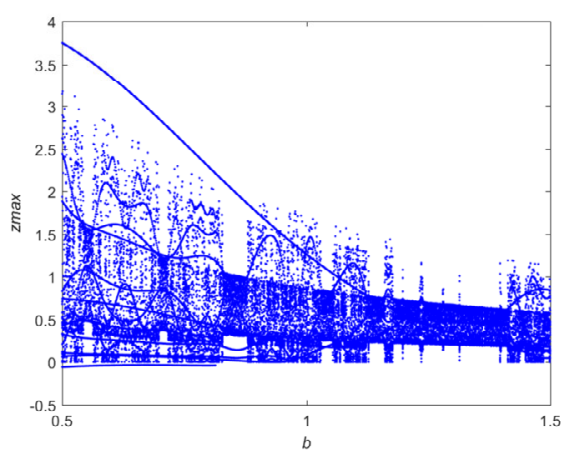

(b)

Figure 3 Bifurcations of (3) for (a) $0 \leq a \leq 0.5$ and $b=1$ (b) $a=0.2$ and $0.5 \leq b \leq 1.5$ 


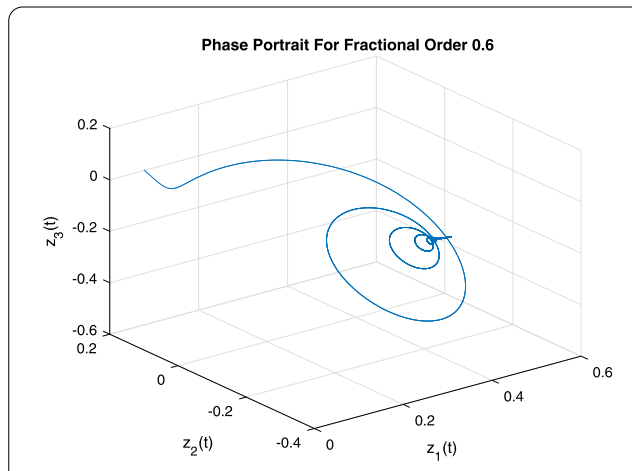

(a)

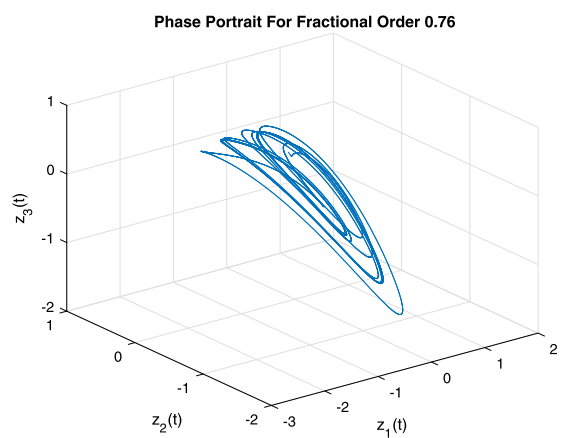

(c)

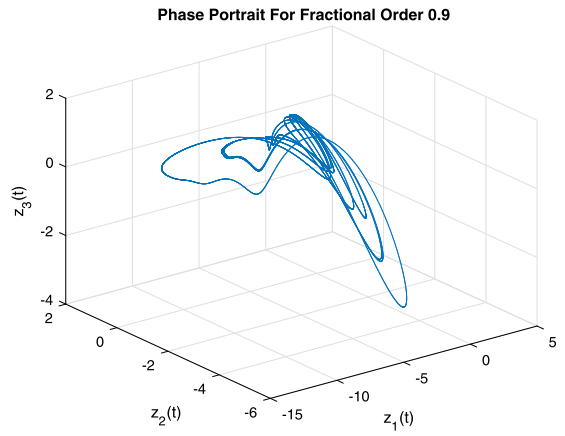

(e)

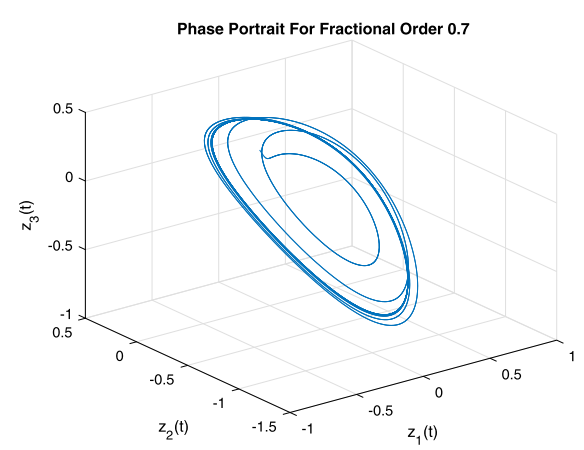

(b)

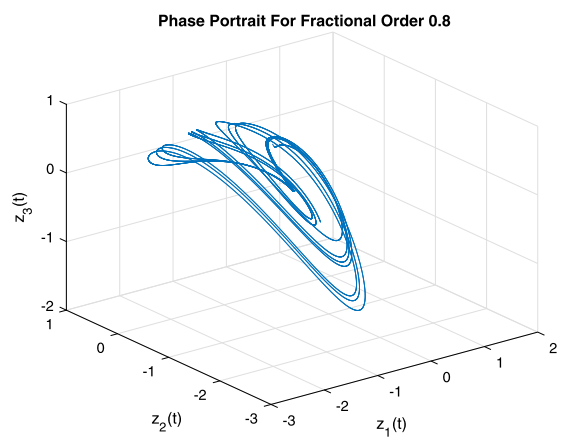

(d)

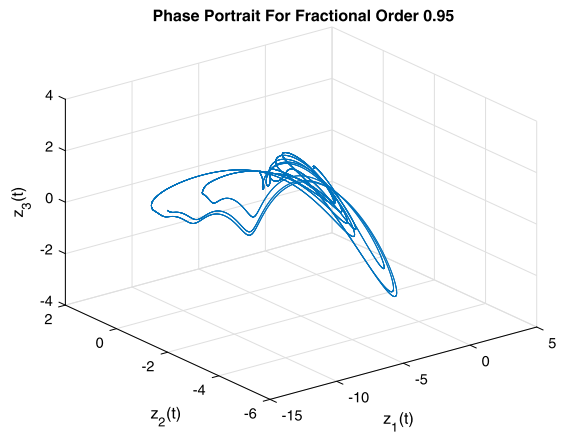

(f)

Figure 4 Attractor of (3) for q (a) 0.6 (b) 0.7 (c) 0.76 (d) 0.8 (e) 0.9 (f) 0.95

By [52], we have a necessary condition on fractional order to have chaos is $q>$ $\frac{2}{\pi} \arctan \left(\frac{|\operatorname{Im}(\mu)|}{\operatorname{Re}(\mu)}\right)$. Therefore the smallest $q$ for (3) to have chaos is 0.76 . Different q plots of (3) are in Fig. 4.

In Fig. 5(a) the Lyapunov dynamics of (3) for q between 0.8 to 1 is shown. From the figure we observe more chaos in the system for fractional order 0.86 and above. In Fig. 5(b) the bifurcations of (3) for order between the 0.8 to 1 range are shown. On crossing a value, the increase in the density of points shows the increasing bifurcations undergoing in the system. 


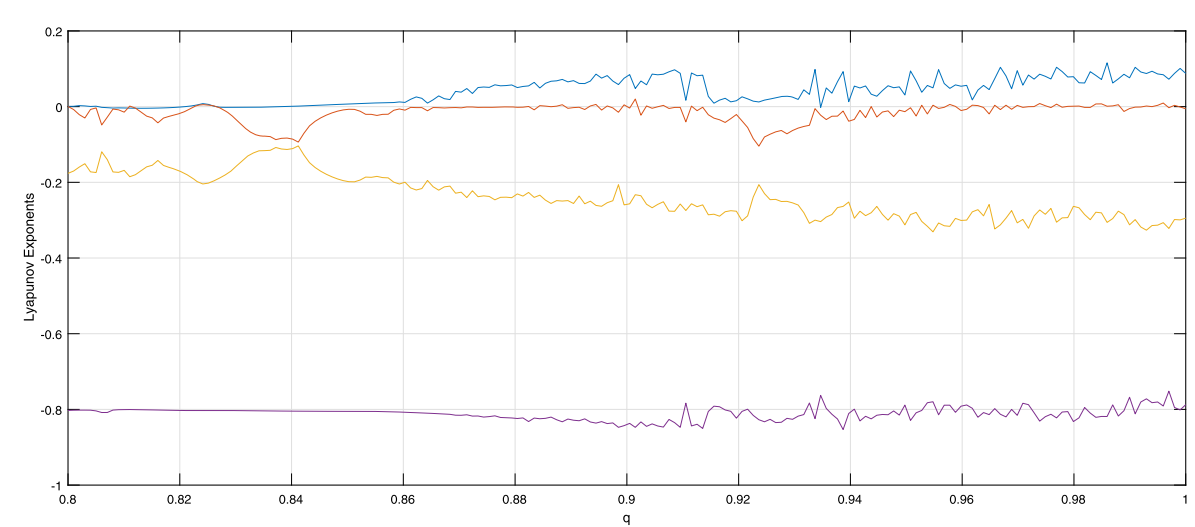

(a)

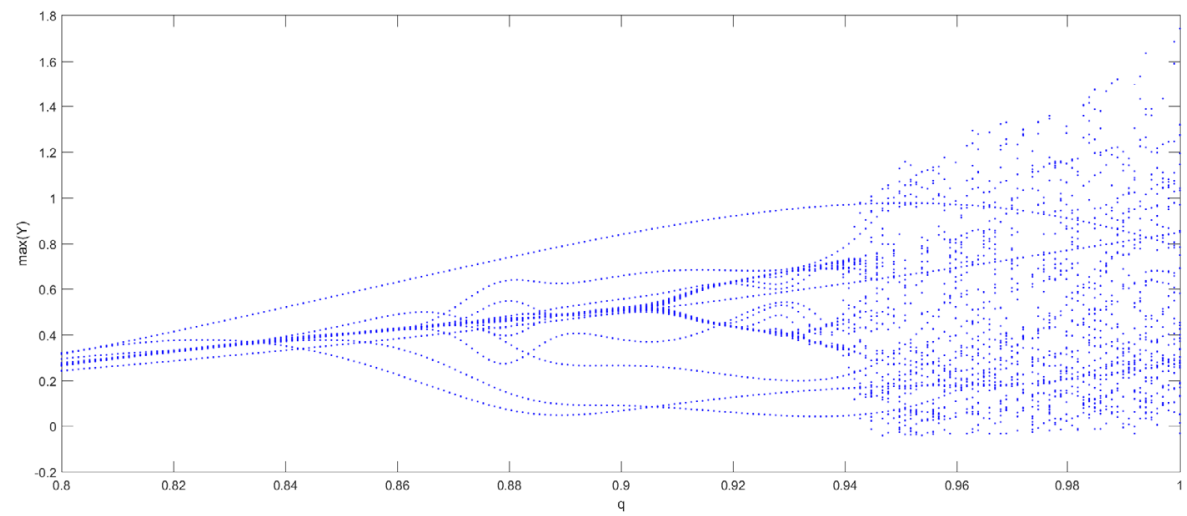

(b)

Figure 5 Lyapunov dynamics and bifurcations for varying $q$

\section{Triple compound combination anti-synchronization}

We first consider the six parallel systems of (3) defined as

$$
\begin{aligned}
& D^{q} v_{11}=v_{12}, \\
& D^{q} v_{12}=v_{13}, \\
& D^{q} v_{13}=v_{14}, \\
& D^{q} v_{14}=-a v_{11}-v_{12}-v_{14}+P b v_{11} v_{13},
\end{aligned}
$$

summarized in the form of a table as follows: triple compound combination antisynchronization is achieved here between system (3) and six systems listed in Table 2 [18]. The phase portraits of such systems are parallel shown in Fig. 6.

In presence of uncertainty and disturbances consider two leader systems and six follower systems. Figure 7 shows the plots of a leader system. 
Table 2 Parallel systems for $a=0.2, b=1$ and $q=0.95$

\begin{tabular}{lll}
\hline Parallel System No. & P & I.C. \\
\hline I & 2 & $(0,0.2,0.2,0)$ \\
II & 3 & $(0,0.3,0.3,0)$ \\
III & 1 & $(0,0.1,0.1,0)$ \\
IV & 0.5 & $(0,0.05,0.05,0)$ \\
V & 0.33 & $(0,0.033,0.033,0)$ \\
VI & 1.5 & $(0,0.15,0.15,0)$ \\
\hline
\end{tabular}

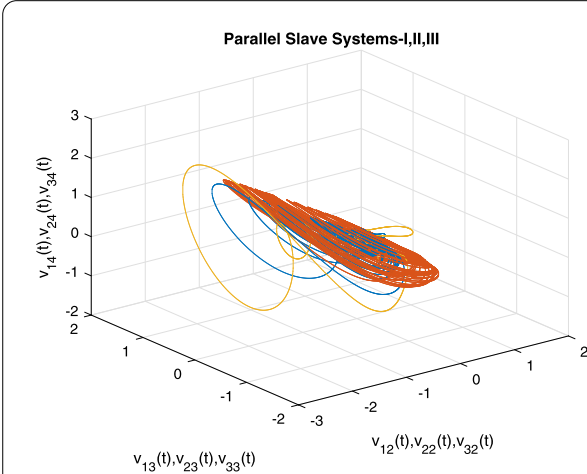

(a)

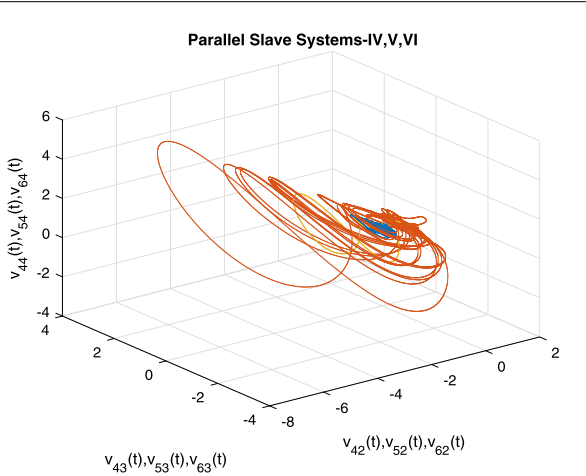

(b)

Figure 6 Parallel phase portraits

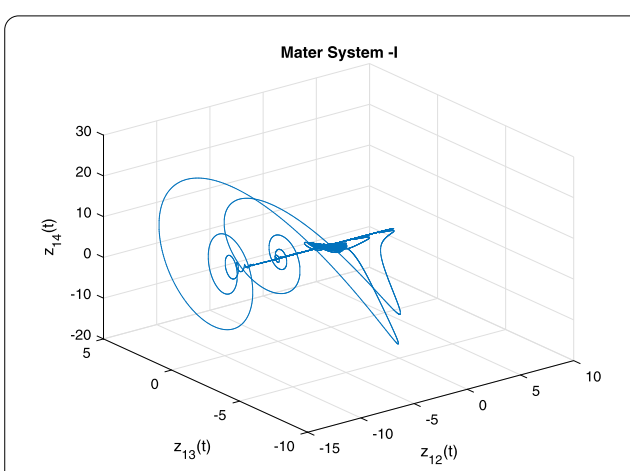

(a)

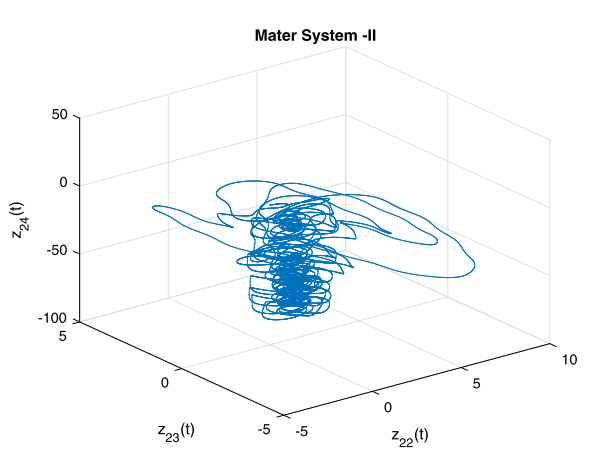

(b)

Figure 7 Chaotic attractor of the master systems

\section{Leader I:}

$$
\begin{aligned}
& D^{q} z_{11}=z_{12}+\triangle g_{1}+\psi_{1}, \\
& D^{q} z_{12}=z_{13}+\triangle g_{2}+\psi_{2}, \\
& D^{q} z_{13}=z_{14}+\triangle g_{3}+\psi_{3}, \\
& D^{q} z_{14}=-a z_{11}-z_{12}-z_{14}+b z_{11} z_{13}+\Delta g_{4}+\psi_{4} .
\end{aligned}
$$

Here, $a=0.2, b=1, \Delta g_{1}=0, \Delta g_{2}=0.7 \sin (t), \Delta g_{3}=0, \Delta g_{4}=0$, and I.C. $(0,0.1,0.1,0), q=$ 0.95 and $\psi_{1}, \psi_{2}, \psi_{3}, \psi_{4}$ are controllers. 
Leader II:

$$
\begin{aligned}
& D^{q} z_{21}=z_{22}+\Delta h_{1}+\phi_{1}, \\
& D^{q} z_{22}=z_{23}+\Delta h_{2}+\phi_{2}, \\
& D^{q} z_{23}=z_{24}+\Delta h_{3}+\phi_{3}, \\
& D^{q} z_{24}=-a z_{21}-z_{22}-z_{24}+b z_{21} z_{23}+\Delta h_{4}+\phi_{4} .
\end{aligned}
$$

Here, $a=0.2, b=1, \Delta h_{1}=0, \Delta h_{2}=\sin \left(\pi z_{23}\right), \Delta g_{3}=0.2 t, \Delta g_{4}=0$, and I.C. $(0,0.1,0.1,0)$, $q=0.95$ and $\phi_{1}, \phi_{2}, \phi_{3}, \phi_{4}$ are controllers.

Follower I:

$$
\begin{aligned}
& D^{q} w_{11}=w_{12}, \\
& D^{q} w_{12}=w_{13}, \\
& D^{q} w_{13}=w_{14}, \\
& D^{q} w_{14}=-a w_{11}-w_{12}-w_{14}+b w_{11} w_{13} .
\end{aligned}
$$

Here, $a=0.2, b=1$, and I.C. $\left(w_{11}(0), w_{12}(0), w_{13}(0), w_{14}(0)\right)=(0,0.1,0.1,0)$ with $q=0.95$.

Follower II:

$$
\begin{aligned}
& D^{q} w_{21}=w_{22}, \\
& D^{q} w_{22}=w_{23}, \\
& D^{q} w_{23}=w_{24}, \\
& D^{q} w_{24}=-a w_{21}-w_{22}-w_{24}+0.5 b w_{21} w_{23} .
\end{aligned}
$$

Here, $a=0.2, b=1$, and I.C. $(0,0.05,0.05,0)$ with $q=0.95$.

Follower III:

$$
\begin{aligned}
& D^{q} w_{31}=w_{32}, \\
& D^{q} w_{32}=w_{33}, \\
& D^{q} w_{33}=w_{34}, \\
& D^{q} w_{34}=-a w_{31}-w_{32}-w_{34}+2 b w_{31} w_{33} .
\end{aligned}
$$

Here, $a=0.2, b=1$, and I.C. $(0,0.2,0.2,0)$ with $q=0.95$.

Follower IV:

$$
\begin{aligned}
& D^{q} w_{41}=w_{42}, \\
& D^{q} w_{42}=w_{43}, \\
& D^{q} w_{43}=w_{44}, \\
& D^{q} w_{44}=-a w_{41}-w_{42}-w_{44}+1.5 b w_{41} w_{43} .
\end{aligned}
$$

Here, $a=0.2, b=1$, and I.C. $(0,0.15,0.15,0)$ with $q=0.95$. 
Follower V:

$$
\begin{aligned}
& D^{q} w_{51}=w_{52}, \\
& D^{q} w_{52}=w_{53}, \\
& D^{q} w_{53}=w_{54}, \\
& D^{q} w_{54}=-a w_{51}-w_{52}-w_{54}+0.33 b w_{51} w_{53} .
\end{aligned}
$$

Here, $a=0.2, b=1$, and I.C. $(0,0.033,0.033,0)$ with $q=0.95$.

Follower VI:

$$
\begin{aligned}
& D^{q} w_{61}=w_{62}, \\
& D^{q} w_{62}=w_{63}, \\
& D^{q} w_{63}=w_{64}, \\
& D^{q} w_{64}=-a w_{61}-w_{62}-w_{64}+3 b w_{61} w_{63} .
\end{aligned}
$$

Here, $a=0.2, b=1$, and I.C. $(0,0.3,0.3,0)$ with $q=0.95$.

The anti-synchronization error is as follows:

$$
\begin{aligned}
& e_{1}=\left(z_{11}+z_{21}\right)+\left(w_{11}+w_{21}\right)\left(w_{31}+w_{41}\right)\left(w_{51}+w_{61}\right), \\
& e_{2}=\left(z_{12}+z_{22}\right)+\left(w_{12}+w_{22}\right)\left(w_{32}+w_{42}\right)\left(w_{52}+w_{62}\right), \\
& e_{3}=\left(z_{13}+z_{23}\right)+\left(w_{13}+w_{23}\right)\left(w_{33}+w_{43}\right)\left(w_{53}+w_{63}\right), \\
& e_{4}=\left(z_{14}+z_{24}\right)+\left(w_{14}+w_{24}\right)\left(w_{34}+w_{44}\right)\left(w_{54}+w_{64}\right) .
\end{aligned}
$$

Differentiating (13), from (5)-(12) we have:

$$
\begin{aligned}
D^{q} e_{1}= & \left(z_{12}+z_{22}+\psi_{1}+\phi_{1}\right)+\left(w_{12}+w_{22}\right)\left(w_{31}+w_{41}\right)\left(w_{51}+w_{61}\right) \\
& +\left(w_{11}+w_{21}\right)\left(w_{32}+w_{42}\right) \\
& \times\left(w_{51}+w_{61}\right)+\left(w_{11}+w_{21}\right)\left(w_{31}+w_{41}\right)\left(w_{52}+w_{62}\right), \\
D^{q} e_{2}= & \left(z_{13}+0.7 \sin (t)+z_{23}+\sin \left(\pi z_{23}\right)+\psi_{2}+\phi_{2}\right) \\
& +\left(w_{13}+w_{23}\right)\left(w_{32}+w_{42}\right)\left(w_{52}+w_{62}\right) \\
& +\left(w_{12}+w_{22}\right)\left(w_{33}+w_{43}\right)\left(w_{52}+w_{62}\right)+\left(w_{12}+w_{22}\right)\left(w_{32}+w_{42}\right)\left(w_{53}+w_{63}\right), \\
D^{q} e_{3}= & \left(z_{14}+0.2 t+z_{24}+\psi_{3}+\phi_{3}\right)+\left(w_{14}+w_{24}\right)\left(w_{33}+w_{43}\right)\left(w_{53}+w_{63}\right) \\
& +\left(w_{13}+w_{23}\right)\left(w_{34}+w_{44}\right)\left(w_{53}+w_{63}\right)+\left(w_{13}+w_{23}\right)\left(w_{33}+w_{43}\right)\left(w_{54}+w_{64}\right), \\
D^{q} e_{4}= & \left(-a z_{11}-z_{12}-z_{14}+2 b z_{11} z_{13}-a z_{21}-z_{22}-z_{24}+3 b z_{21} z_{23}+\psi_{4}+\phi_{4}\right) \\
& +\left(-a w_{11}-w_{12}-w_{14}+b w_{11} w_{13}-a w_{21}-w_{22}-w_{24}+0.5 b w_{21} w_{23}\right) \\
& \times\left(w_{34}+w_{44}\right)\left(w_{54}+w_{64}\right) \\
& +\left(w_{14}+w_{24}\right) \\
& \times\left(-a w_{31}-w_{32}-w_{34}+2 b w_{31} w_{33}-a w_{41}-w_{42}-w_{44}+1.5 b w_{41} w_{43}\right)
\end{aligned}
$$




$$
\begin{aligned}
& \times\left(w_{54}+w_{64}\right)+\left(w_{14}+w_{24}\right)\left(w_{34}+w_{44}\right) \\
& \times\left(-a w_{51}-w_{52}-w_{54}+0.33 b w_{51} w_{53}-a w_{61}-w_{62}-w_{64}+3 b w_{61} w_{63}\right) .
\end{aligned}
$$

\subsection{Via nonlinear control}

Choosing controllers as follows:

$$
\begin{aligned}
\psi_{1}= & -z_{12}-\left(w_{12}+w_{22}\right)\left(w_{31}+w_{41}\right)\left(w_{51}+w_{61}\right) \\
& -\left(w_{11}+w_{21}\right)\left(w_{31}+w_{41}\right)\left(w_{52}+w_{62}\right), \\
\psi_{2}= & -z_{13}-0.7 \sin (t)-\left(w_{13}+w_{23}\right)\left(w_{32}+w_{42}\right)\left(w_{52}+w_{62}\right) \\
& -\left(w_{12}+w_{22}\right)\left(w_{32}+w_{42}\right)\left(w_{53}+w_{63}\right), \\
\psi_{3}= & -z_{14}-\left(w_{14}+w_{24}\right)\left(w_{33}+w_{43}\right)\left(w_{53}+w_{63}\right) \\
& -\left(w_{13}+w_{23}\right)\left(w_{33}+w_{43}\right)\left(w_{54}+w_{64}\right) \\
\psi_{4}= & \left(a z_{11}+z_{12}+z_{14}-2 b z_{11} z_{13}\right) \\
& -\left(-a w_{11}-w_{12}-w_{14}+b w_{11} w_{13}-a w_{21}-w_{22}-w_{24}\right. \\
& \left.+0.5 b w_{21} w_{23}\right)\left(w_{34}+w_{44}\right)\left(w_{54}+w_{64}\right)-4 e_{4}, \\
\phi_{1}= & -z_{22}-\left(w_{11}+w_{21}\right)\left(w_{32}+w_{42}\right)\left(w_{51}+w_{61}\right)-e_{1}, \\
\phi_{2}= & -z_{23}-\sin \left(\pi z_{23}\right)-\left(w_{12}+w_{22}\right)\left(w_{33}+w_{43}\right)\left(w_{52}+w_{62}\right)-2 e_{2}, \\
\phi_{3}= & -z_{24}-0.2 t-\left(w_{13}+w_{23}\right)\left(w_{34}+w_{44}\right)\left(w_{53}+w_{63}\right)-3 e_{3}, \\
\phi_{4}= & \left(a z_{21}+z_{22}+z_{24}-3 b z_{21} z_{23}\right) \\
& -\left(w_{14}+w_{24}\right)\left(-a w_{31}-w_{32}-w_{34}+2 b w_{31} w_{33}-a w_{41}-w_{42}\right. \\
& \left.-w_{44}+1.5 b w_{41} w_{43}\right)\left(w_{54}+w_{64}\right)-\left(w_{14}+w_{24}\right)\left(w_{34}+w_{44}\right)\left(-a w_{51}-w_{52}-w_{54}\right. \\
& \left.0.33 w_{51} w_{53}-a w_{61}-w_{62}-w_{64}+3 b w_{61} w_{63}\right) . \\
&
\end{aligned}
$$

Substituting (15) into (14), we get

$$
\begin{aligned}
& D^{q} e_{1}=-e_{1}, \\
& D^{q} e_{2}=-2 e_{2}, \\
& D^{q} e_{3}=-3 e_{3}, \\
& D^{q} e_{4}=-4 e_{4} .
\end{aligned}
$$

Define the Lyapunov function

$$
\begin{aligned}
& V(e(t))=\frac{1}{2}\left(e_{1}^{2}+e_{2}^{2}+e_{3}^{2}+e_{4}^{2}\right) \\
& \begin{aligned}
D^{q} V(e(t)) & \leq-e_{1}{ }^{2}-2 e_{2}{ }^{2}-3 e_{3}{ }^{2}-4 e_{4}{ }^{2} \\
& \Rightarrow D^{q} V(e(t)) \quad \text { is negative definite. }
\end{aligned}
\end{aligned}
$$

Lyapunov stability theory proves $\left\|e_{i}\right\| \rightarrow 0$ as $t \rightarrow \infty$, implying synchronization. 


\subsection{Via adaptive SMC}

Assuming bounded uncertainties and disturbances, we have $\left|\triangle g_{i}\right| \leq a_{i}$ and $\left|\triangle h_{i}\right| \leq b_{i}$ for positive real numbers $a_{i}$ and $b_{i}$, and $\hat{a}_{i}$ and $\hat{b}_{i}$ are estimated values of $a_{i}$ and $b_{i}$.

Choosing the sliding surface so as to minimize the error:

$$
s_{i}(t)=D^{q-1} e_{i}(t)+k_{i} \int_{0}^{t} e_{i}(\xi) d \xi
$$

For (14) to be at (16), we must have

$$
s_{i}(t)=0, \quad \dot{s}_{i}(t)=0 .
$$

Taking Caputo's derivative of (17)

$$
\dot{s}_{i}(t)=D^{q} e_{i}(t)+k_{i} e_{i}(t)
$$

then from (17) we have

$$
D^{q} e_{i}(t)=-k_{i} e_{i}(t) .
$$

The error dynamical system is stable by the Matignon theorem [33]. Using SMC theory, we design controllers:

$$
\begin{aligned}
\psi_{1}= & -z_{12}-\left(w_{12}+w_{22}\right)\left(w_{31}+w_{41}\right)\left(w_{51}+w_{61}\right) \\
& -\left(w_{11}+w_{21}\right)\left(w_{31}+w_{41}\right)\left(w_{52}+w_{62}\right), \\
\psi_{2}= & -z_{13}-\left(w_{13}+w_{23}\right)\left(w_{32}+w_{42}\right)\left(w_{52}+w_{62}\right) \\
& -\left(w_{12}+w_{22}\right)\left(w_{32}+w_{42}\right)\left(w_{53}+w_{63}\right), \\
\psi_{3}= & -z_{14}-\left(w_{14}+w_{24}\right)\left(w_{33}+w_{43}\right)\left(w_{53}+w_{63}\right) \\
& -\left(w_{13}+w_{23}\right)\left(w_{33}+w_{43}\right)\left(w_{54}+w_{64}\right), \\
\psi_{4}= & \left(a z_{11}+z_{12}+z_{14}-2 b z_{11} z_{13}\right) \\
& -\left(-a w_{11}-w_{12}-w_{14}+b w_{11} w_{13}-a w_{21}-w_{22}-w_{24}\right. \\
& \left.+0.5 b w_{21} w_{23}\right)\left(w_{34}+w_{44}\right)\left(w_{54}+w_{64}\right) \\
& -\left(w_{14}+w_{24}\right)\left(w_{34}+w_{44}\right)\left(-a w_{51}-w_{52}-w_{54}\right. \\
& \left.+0.33 b w_{51} w_{53}-a w_{61}-w_{62}-w_{64}+3 b w_{61} w_{63}\right), \\
\phi_{1}= & \left.-z_{22}-\left(w_{11}+w_{21}\right)\left(w_{32}+w_{42}\right)\left(w_{51}+w_{61}\right)-e_{1}-\left(\hat{a_{1}}+\hat{b_{1}}+\gamma_{1}\right) \operatorname{sign} s_{1}\right), \\
\phi_{2}= & \left.-z_{23}-\left(w_{12}+w_{22}\right)\left(w_{33}+w_{43}\right)\left(w_{52}+w_{62}\right)-2 e_{2}-\left(\hat{a_{2}}+\hat{b}_{2}+\gamma_{2}\right) \operatorname{sign} s_{2}\right), \\
\phi_{3}= & \left.-z_{24}-\left(w_{13}+w_{23}\right)\left(w_{34}+w_{44}\right)\left(w_{53}+w_{63}\right)-3 e_{3}-\left(\hat{a_{3}}+\hat{b_{3}}+\gamma_{3}\right) \operatorname{sign} s_{3}\right), \\
\phi_{4}= & \left(a z_{21}+z_{22}+z_{24}-3 b z_{21} z_{23}\right) \\
& -\left(w_{14}+w_{24}\right)\left(-a w_{31}-w_{32}-w_{34}+2 b w_{31} w_{33}-a w_{41}\right. \\
& \left.\left.-w_{42}-w_{44}+1.5 b w_{41} w_{43}\right)\left(w_{54}+w_{64}\right)-4 e_{4}-\left(\hat{a_{4}}+\hat{b_{4}}+\gamma_{4}\right) \operatorname{sign} s_{4}\right)
\end{aligned}
$$

for signum function $\operatorname{sign}(\cdot)$ and positive real numbers $\gamma_{i}$. 
Adaptive parameter update conditions are as follows:

$$
\dot{\hat{a}}_{i}=p_{i}\left|s_{i}\right|, \quad \dot{\hat{b}}_{i}=q_{i}\left|s_{i}\right|,
$$

where $p_{i}$ and $q_{i}$ are positive constants.

Theorem 8.1 Stability and triple compound combination anti-synchronization is achieved in presence of uncertainties and disturbances between leader systems (10)-(11) and follower systems (12)-(17) with controllers and update conditions as (25) and (26).

Proof Lyapunov's direct method [57] is used to achieve stability between leader and follower systems. Considering V to be a positive definite function with negative definite derivative, asymptotic vanishing of error is proved.

Let

$$
V=V_{1}+V_{2}+V_{3}+V_{4}
$$

where

$$
\begin{aligned}
& V_{1}=\frac{1}{2} s_{1}^{2}+\frac{1}{2 p_{1}}\left(\hat{a}_{1}-a_{1}\right)^{2}+\frac{1}{2 q_{1}}\left(\hat{b}_{1}-b_{1}\right)^{2}, \\
& V_{2}=\frac{1}{2} s_{2}^{2}+\frac{1}{2 p_{2}}\left(\hat{a}_{2}-a_{2}\right)^{2}+\frac{1}{2 q_{2}}\left(\hat{b}_{2}-b_{2}\right)^{2}, \\
& V_{3}=\frac{1}{2} s_{3}^{2}+\frac{1}{2 p_{3}}\left(\hat{a}_{3}-a_{3}\right)^{2}+\frac{1}{2 q_{3}}\left(\hat{b}_{3}-b_{3}\right)^{2}, \\
& V_{4}=\frac{1}{2} s_{4}^{2}+\frac{1}{2 p_{4}}\left(\hat{a}_{4}-a_{4}\right)^{2}+\frac{1}{2 q_{4}}\left(\hat{b}_{4}-b_{4}\right)^{2} .
\end{aligned}
$$

Differentiating (23):

$$
\begin{aligned}
& \dot{V}_{1}=s_{1} \dot{s_{1}}+\frac{1}{p_{1}}\left(\hat{a}_{1}-a_{1}\right) \dot{\hat{a}}_{1}+\frac{1}{q_{1}}\left(\hat{b}_{1}-b_{1}\right) \dot{\hat{b}}_{1}, \\
& \dot{V}_{2}=s_{2} \dot{s_{2}}+\frac{1}{p_{2}}\left(\hat{a}_{2}-a_{2}\right) \dot{\hat{a}}_{2}+\frac{1}{q_{2}}\left(\hat{b}_{2}-b_{2}\right) \dot{\hat{b}}_{2}, \\
& \dot{V}_{3}=s_{3} \dot{s_{3}}+\frac{1}{p_{3}}\left(\hat{a}_{3}-a_{3}\right) \dot{\hat{a}}_{3}+\frac{1}{q_{3}}\left(\hat{b}_{3}-b_{3}\right) \dot{\hat{b}}_{3}, \\
& \dot{V}_{4}=s_{4} \dot{s}_{4}+\frac{1}{p_{4}}\left(\hat{a}_{4}-a_{4}\right) \dot{\hat{a}}_{4}+\frac{1}{q_{4}}\left(\hat{b}_{4}-b_{4}\right) \dot{\hat{b}}_{4} .
\end{aligned}
$$

From (18), we have

$$
\begin{aligned}
& \dot{V}_{1}=s_{1}\left(D^{q} e_{1}+k_{1} e_{1}\right)+\frac{1}{p_{1}}\left(\hat{a}_{1}-a_{1}\right) \dot{\hat{a}}_{1}+\frac{1}{q_{1}}\left(\hat{b}_{1}-b_{1}\right) \dot{\hat{b}}_{1}, \\
& \dot{V}_{2}=s_{2}\left(D^{q} e_{2}+k_{2} e_{2}\right)+\frac{1}{p_{2}}\left(\hat{a}_{2}-a_{2}\right) \dot{\hat{a}}_{2}+\frac{1}{q_{2}}\left(\hat{b}_{2}-b_{2}\right) \dot{\hat{b}}_{2}, \\
& \dot{V}_{3}=s_{3}\left(D^{q} e_{3}+k_{3} e_{3}\right)+\frac{1}{p_{3}}\left(\hat{a}_{3}-a_{3}\right) \dot{\hat{a}}_{3}+\frac{1}{q_{3}}\left(\hat{b}_{3}-b_{3}\right) \dot{\hat{b}}_{3}, \\
& \dot{V}_{4}=s_{4}\left(D^{q} e_{4}+k_{4} e_{4}\right)+\frac{1}{p_{4}}\left(\hat{a}_{4}-a_{4}\right) \dot{\hat{a}}_{4}+\frac{1}{q_{4}}\left(\hat{b}_{4}-b_{4}\right) \dot{\hat{b}}_{4} .
\end{aligned}
$$


Substituting $D^{q} e_{i}, \dot{\hat{a}}_{i}$, and $\dot{\hat{b}}_{i}$ in (25), we obtain

$$
\begin{aligned}
\dot{V}_{i} & =s_{1}\left[\triangle g_{i}-\Delta h_{i}-\left(\hat{a}_{i}+\hat{b}_{i}+\gamma_{i}\right) \operatorname{sign} s_{i}\right]+\left(\hat{a}_{i}-a_{i}\right)\left|s_{i}\right|+\left(\hat{b}_{i}-b_{i}\right)\left|s_{i}\right| \\
& \leq\left(\left|\triangle g_{i}\right|+\left|\triangle h_{i}\right|\right)\left|s_{i}\right|-\left(\hat{a}_{i}+\hat{b}_{i}+\gamma_{i}\right)\left|\operatorname{sign} s_{i}\right|+\left(\hat{a}_{i}-a_{i}\right)\left|s_{i}\right|+\left(\hat{b}_{i}-b_{i}\right)\left|s_{i}\right| \\
& <\left(a_{i}+b_{i}\right)\left|s_{i}\right|-\left(\hat{a}_{i}+\hat{b}_{i}+\gamma_{i}\right)\left|\operatorname{sign} s_{i}\right|+\left(\hat{a}_{i}-a_{i}\right)\left|s_{i}\right|+\left(\hat{b}_{i}-b_{i}\right)\left|s_{i}\right| \\
& =-R_{i}\left|s_{i}\right| .
\end{aligned}
$$

Finally,

$$
\begin{aligned}
\dot{V} & =\sum_{i=1}^{4} \dot{V}_{i} \\
& <-\sum_{i=1}^{4}\left(R_{i}\left|s_{i}\right|\right) .
\end{aligned}
$$

Thus $\exists R \geq 0 \in R$

$$
\sum_{i=1}^{4} R_{i}\left|s_{i}\right|>R
$$

then we have

$$
\begin{aligned}
\dot{V} & <-R \sqrt{s_{1}^{2}+s_{2}^{2}+s_{3}^{2}+s_{4}^{2}+s_{5}^{2}} \\
& <0 .
\end{aligned}
$$

Lyapunov stability theory proves $\left\|s_{i}\right\| \rightarrow 0$ as $t \rightarrow \infty$, implying stability and synchronization.

\section{Simulation results}

The efficacy of our synchronization methods is verified using MATLAB software for simulations. The trajectories of the triple compound combination anti-synchronized leader system and follower system using nonlinear control are in Fig. 8. The anti-synchronization error vanishing has been displayed in Fig. 9 for the I.C. $(0,0.5175,0.4825,0)$.

Figure 10 gives the anti-synchronized trajectories using the adaptive SMC method with the error vanishing in Fig. 11. The sliding surfaces tend to zero in Fig. 12 with Fig. 13 giving estimates of bounds of disturbances of leader systems. The I.C. for estimates is $\hat{a}_{i}=\hat{b}_{i}=0.1$ and $\gamma_{1}=2, \gamma_{2}=3, \gamma_{3}=3, \gamma_{4}=4$ respectively. Other parameters and conditions are as in Sect. 8 .

\section{Comparison of synchronization results}

The increasing usage of synchronization methods in various fields leads to discovery of new chaotic systems and new synchronization schemes. The time taken to achieve the synchronization is a very key aspect in this regard. Clearly the lesser the time of synchronization, the more is the efficacy of the designed controllers. Table 3 shows the time taken to attain different types of synchronization by different authors and presents a comparative study of combination and compound synchronizations. 


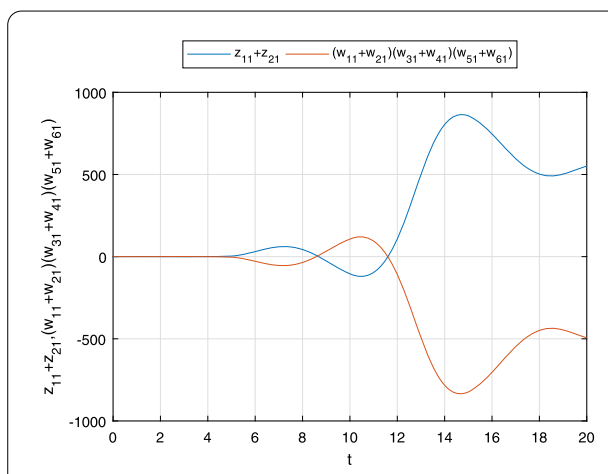

(a)

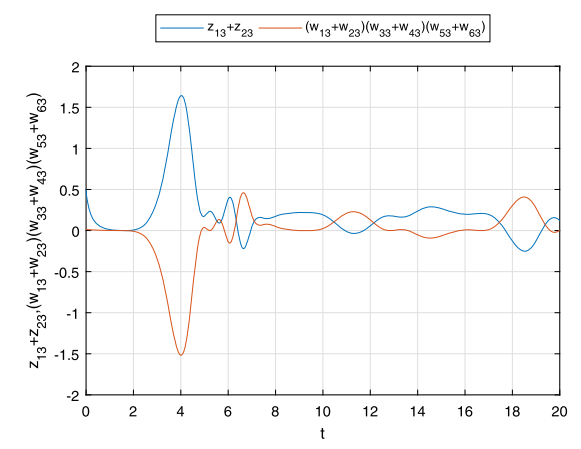

(c)

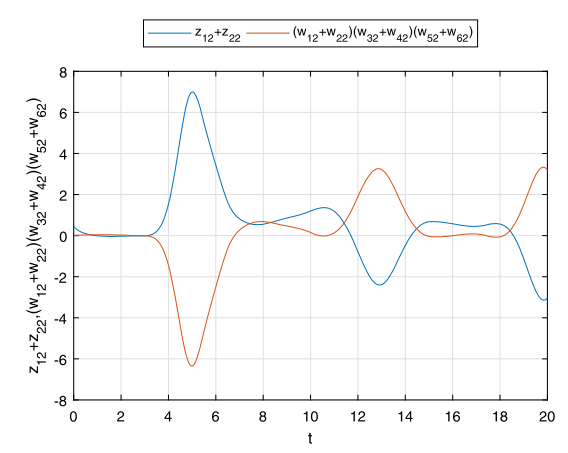

(b)

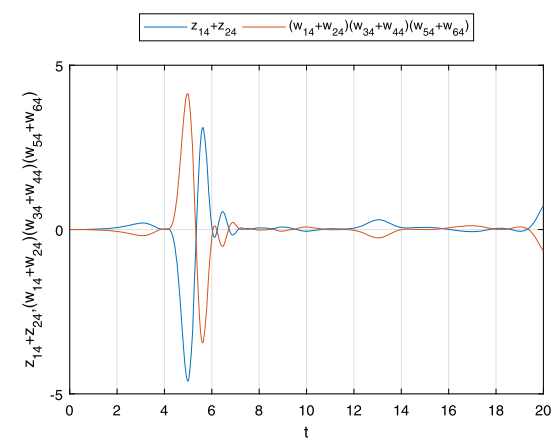

(d)

Figure 8 Nonlinear control synchronized trajectories

Figure 9 Nonlinear control synchronization error

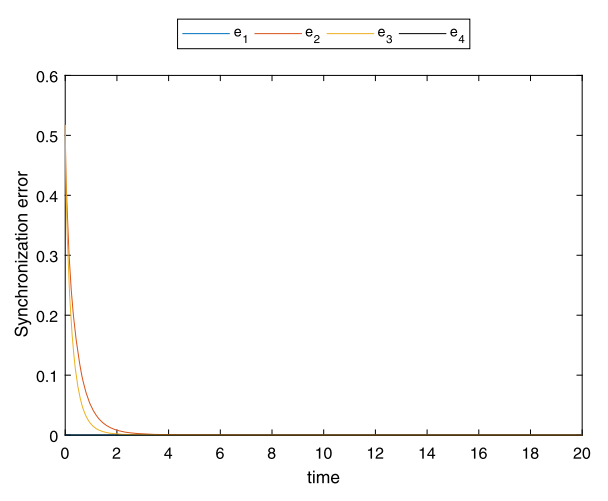

In our paper we have performed triple compound combination synchronization among parallel systems of novel fractional order snap systems. In nonlinear control, error converges to zero at three units of time, and in the adaptive SMC method error approaches zero before two units but oscillates about it. These findings display the efficacy of our designed controllers.

\section{Application in secure communication}

Chaos synchronization, having found application in many different fields such as secure communication $[53,68]$ and image encryption $[46,65]$, has been growing rapidly $[47,48$, 


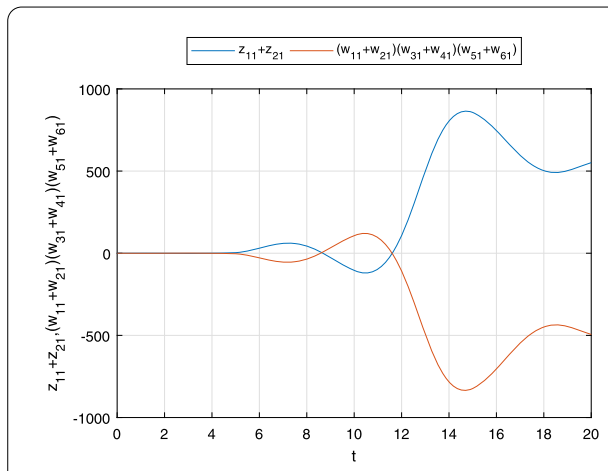

(a)

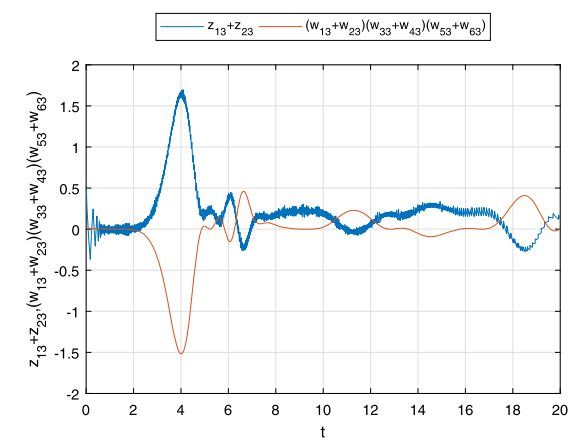

(c)

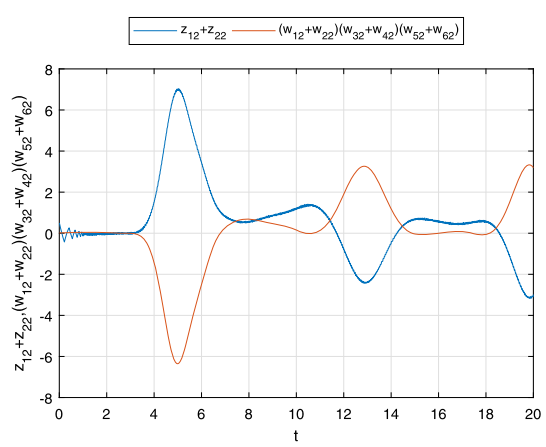

(b)

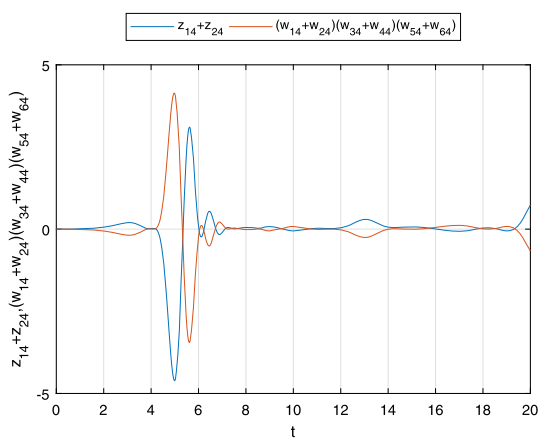

(d)

Figure 10 Adaptive sliding mode control synchronized trajectories

Figure 11 Adaptive sliding mode control synchronization error

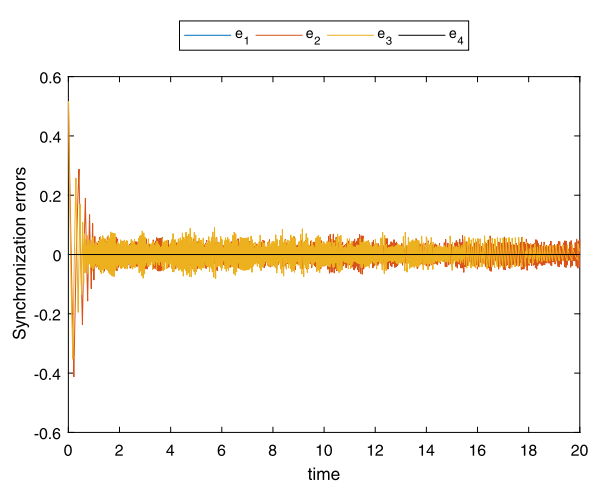

55]. The highly sensitive dependence on I.C. and parameter values makes the information secure from getting hacked by intruders.

Here we use the above anti-synchronization technique to explain its usage in information security. The original signal is mixed with chaotic signals and transmitted from the sending end. Only if prior knowledge of the type of synchronization, I.C., and parameter values are known, the original message can be recovered at the receiving end.

\section{Illustration:}

The original message $x(t)=\sin (7 t)$ is mixed with a combination of chaotic signals $z_{14}+$ $z_{24}$ produced from master systems to obtain the encrypted message as $x_{1}(t)$. On attaining 
Figure 12 Sliding surfaces tending to zero

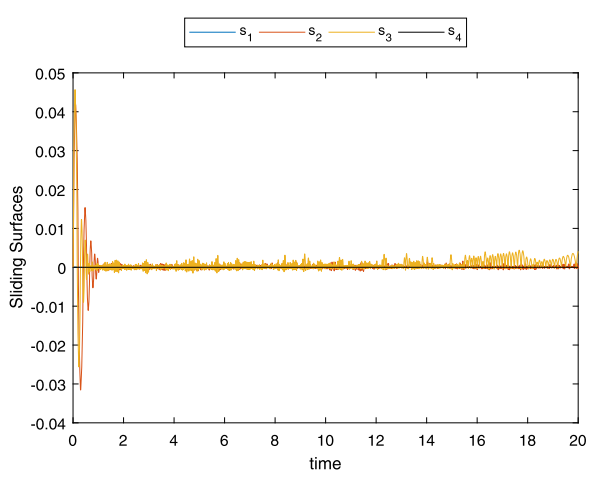

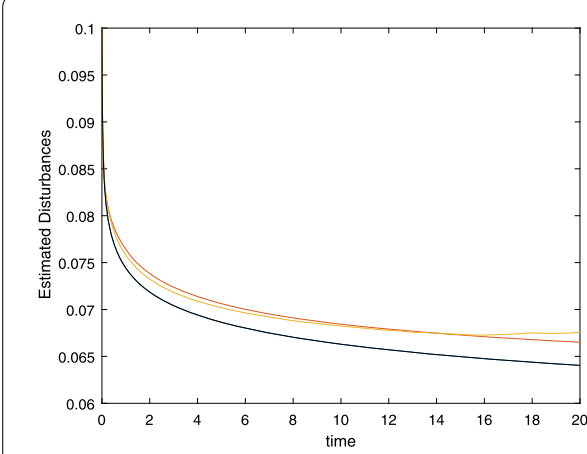

(a)

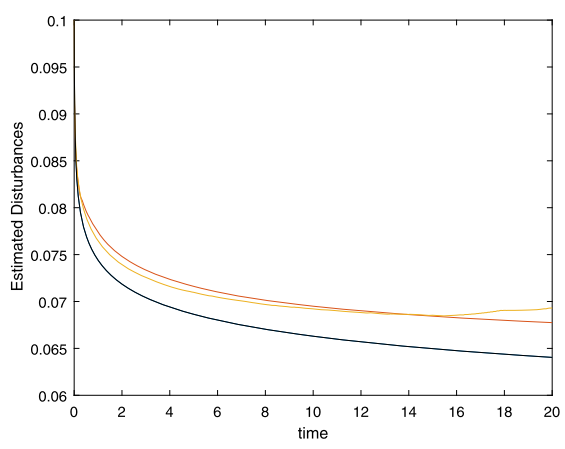

(b)

Figure 13 Bound estimates of uncertainties and disturbances

Table 3 Error convergence time

\begin{tabular}{llc}
\hline Type of synchronization & Authors & Time \\
\hline Combination [24] & Bo, Xiabing, Yun & 3 \\
Compound [51] & Sun, Yi, Shen & 5 \\
Multi-switching compound [39] & Prajapati, Khan, Khattar & 4.5 \\
Double compound [67] & Zhang, Deng & 5 \\
Modified compound [49] & Sun, Li, Wang, Wang & 3 \\
Double compound combination [29] & Mahmoud, Abed-Elhameed & 4 \\
Triple compound [66] & Yadav, Prasad, Srivastava & 5 \\
Dual compound compound [14] & Ibraheem & 4 \\
\hline
\end{tabular}

the right synchronization after applying controllers at the receiver, the original message is recovered viz $x_{2}(t)$. Simulation results are shown in Fig. 14.

\section{Snap system realization}

\subsection{Snap system signal flow graph}

Every state of the snap system (3) is represented as a vertex of the proposed signal flow graph $\vec{H}$ (see Fig. 15, the arrow means its edges have directions). The graph $\vec{H}$ is a weighted graph since each edge has a weight. The weight of the edge $\left(Z_{i}, Z_{j}\right)$ is the impact by which the state variable $Z_{i}$ affects the state variable $Z_{j}$. Then the graph $\vec{H}$ can be 


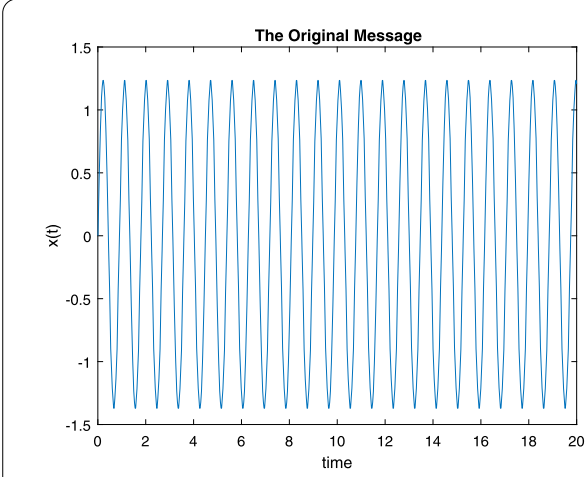

(a)

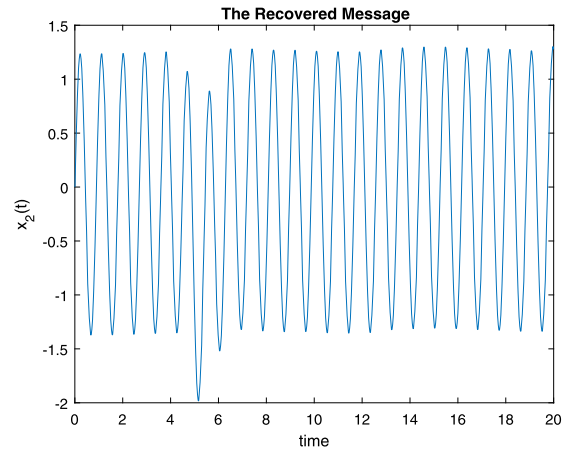

(c)

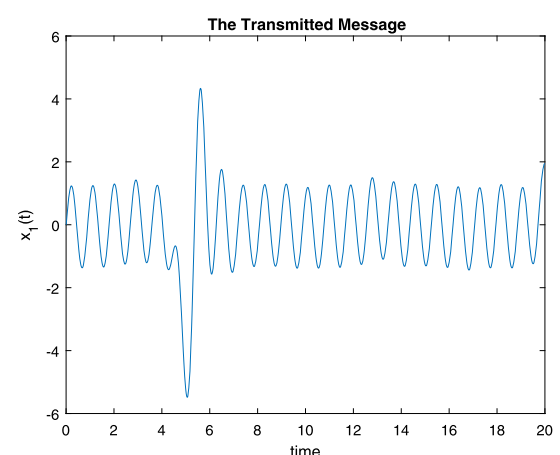

(b)

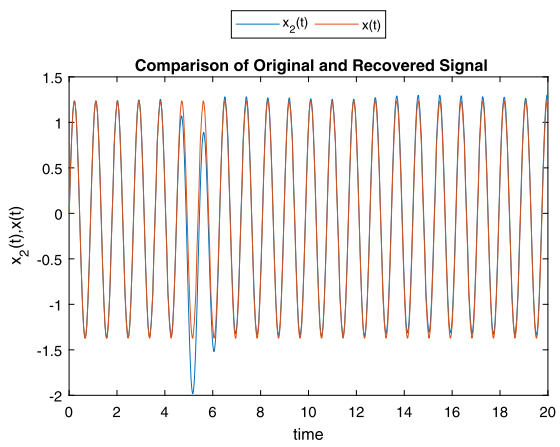

(d)

Figure 14 Illustrative example in secure communication

Figure 15 Snap system signal flow graph $\vec{H}$

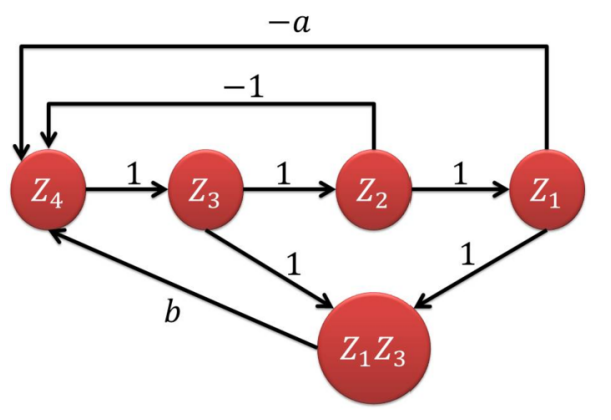

represented by the following weighted adjacency matrix $A(\vec{H})(28)$ :

$$
A(\vec{H})=\begin{gathered}
Z_{1} \\
Z_{2} \\
Z_{3} \\
Z_{4} \\
Z_{1} Z_{3}
\end{gathered}\left[\begin{array}{ccccc}
0 & 0 & 0 & -a & 1 \\
1 & 0 & 0 & -1 & 0 \\
0 & 1 & 0 & 0 & 1 \\
0 & 0 & 1 & -1 & 0 \\
0 & 0 & 0 & b & 0
\end{array}\right] .
$$

For $a=0.2, b=1$, the eigenvalues of $A(\vec{H})$ are $\lambda_{1}=0.82280, \lambda_{2}=0.18418-0.8833 i$, $\lambda_{3}=0.18418+0.8833 i, \lambda_{4}=-1.0956+0.54083 i, \lambda_{5}=-1.0956-0.54083 i$. Then energy $(1)$ 
of the snap system (3) can calculated as

$$
E(H)=\sum_{i=1}^{5}\left|\lambda_{i}\right|=5.071 .
$$

If the directions of the arcs and loops in $\vec{H}$ are omitted, we can get the underlined graph $H$ whose adjacency matrix $A(H)$ (29) can be formed as follows (Note that $A(i, j)=1$ if vertex $i$ is linked with vertex $j$ in $H$ regardless of the direction and $A(i, j)=0$ otherwise.):

$$
A(H)=\begin{gathered}
Z_{1} \\
Z_{2} \\
Z_{3} \\
Z_{4} \\
Z_{1} Z_{3}
\end{gathered}\left[\begin{array}{lllll}
0 & 1 & 0 & 1 & 1 \\
1 & 0 & 1 & 1 & 0 \\
0 & 1 & 0 & 1 & 1 \\
1 & 1 & 1 & 0 & 1 \\
1 & 0 & 1 & 1 & 0
\end{array}\right] .
$$

$A(H)$ represents the structure of the snap system and can help in calculating the energy of the system from the graph theory point of view as follows.

$A(H)$ eigenvalues are $\lambda_{1}=\frac{1}{2} \sqrt{17}+\frac{3}{2}, \lambda_{2}=\frac{3}{2}-\frac{1}{2} \sqrt{17}, \lambda_{3}=-2, \lambda_{4}, \lambda_{5}=0$, then the energy of the snap system can calculated as follows:

$$
E(H)=\sum_{i=1}^{5}\left|\lambda_{i}\right|=2 \sqrt{5}+2 .
$$

\subsection{Snap system electronic circuit realization}

In chaos applications, a very important topic is the hardware realization of mathematical models [28]. We implement an electronic circuit for realizing the studied snap system (3) in this section.

Figure 16 shows the schematic of the designed electronic circuit for the studied snap system (3), which is based on four integrators $(Z 1, \ldots, Z 4)$. All integrators and inverters are constructed using the 741 operational amplifier with Package type DIP-8 and Package manufacturer IPC-2221A/2222.

Equations of the designed electronic circuit of Fig. 16 are as follows:

$$
\begin{aligned}
& Z_{1}=\frac{1}{R_{1} C_{1}} \int \frac{d Z_{1}}{d t} d t, \\
& Z_{2}=\frac{1}{R_{3} C_{2}} \int \frac{d Z_{z}}{d t} d t, \\
& Z_{3}=\frac{1}{R_{5} C_{3}} \int \frac{d Z_{3}}{d t} d t, \\
& Z_{4}=\frac{1}{R_{11} C_{4}} \int \frac{d Z_{1}}{d t} d t,
\end{aligned}
$$

where the symbols $Z 1, Z 2, Z 3$, and $Z 4$ are the voltages of the integrators.

The circuit has been realized via NI MULTISIM 14.0 for $R_{1}=R_{3}=R_{5}=R_{11}=1 \mathrm{k} \Omega, C_{1}=$ $C_{2}=C_{3}=C_{4}=1 \mu \mathrm{F}$. We use five electronic summers $S_{1}, \ldots, S_{5}$, one electronic multiplier 


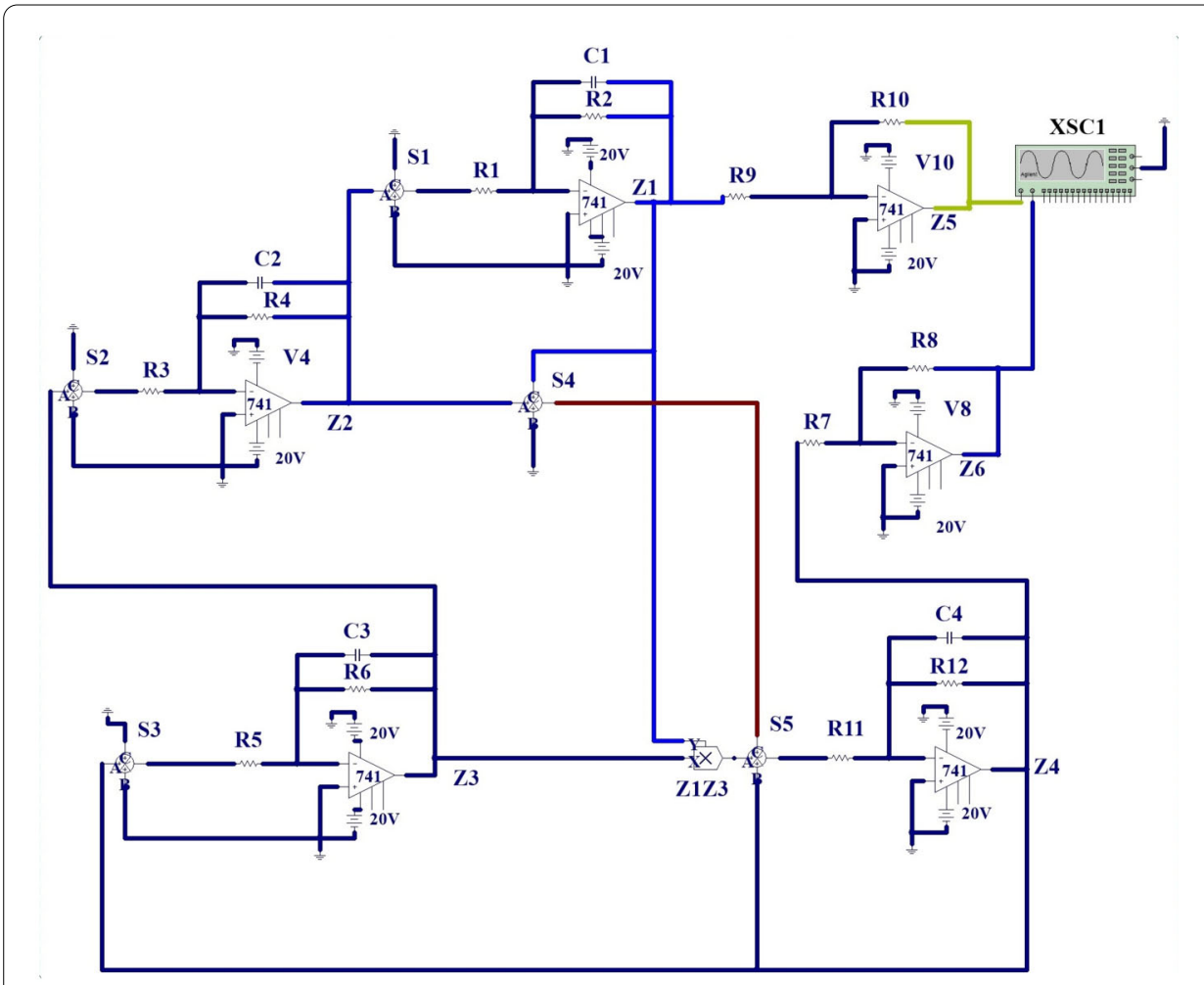

Figure 16 The electronic circuit of system

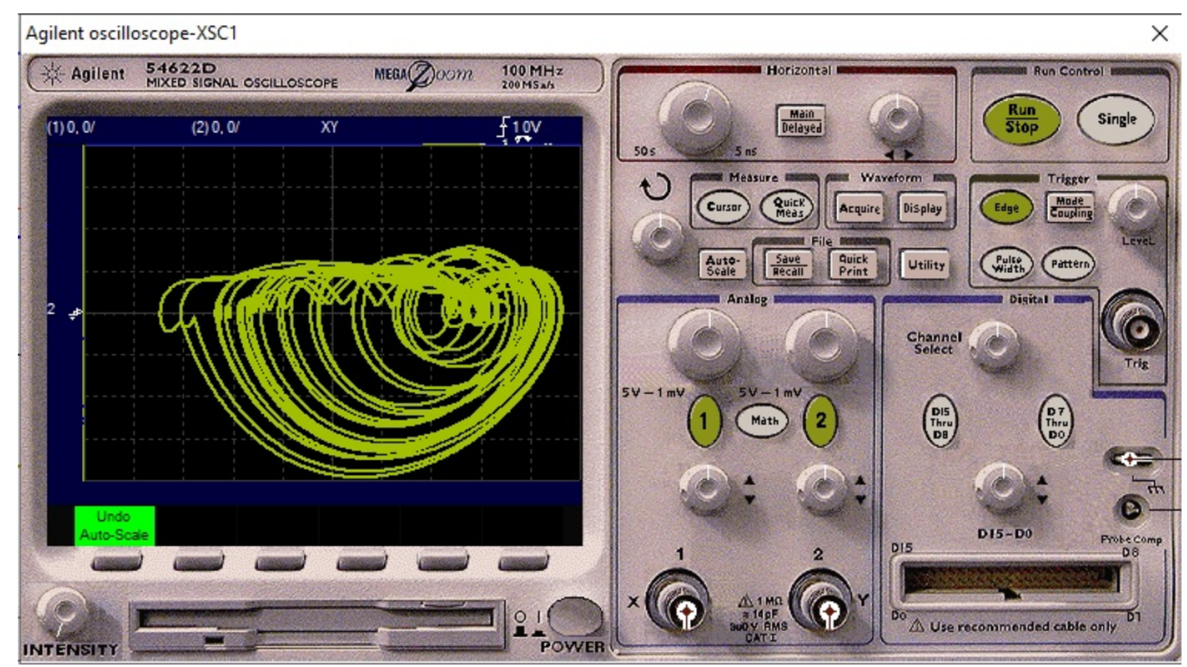

Figure 17 The plot of $Z_{2}$ versus $Z_{1}$ from the electronic circuit

$Z 1 Z 3$, and two inverters $Z 5$ and $Z 6$. An oscilloscope has been used for displaying the measurements and capturing the experimental results.

As a result, the experimental phase portraits of circuit's outputs, for the values of $a, b$ as in the corresponding plots of Fig. 1, are given as shown in Figs. 17, 18, and 19. As predicted, the acquired experimental outputs assure the feasibleness of the studied snap system (3). 

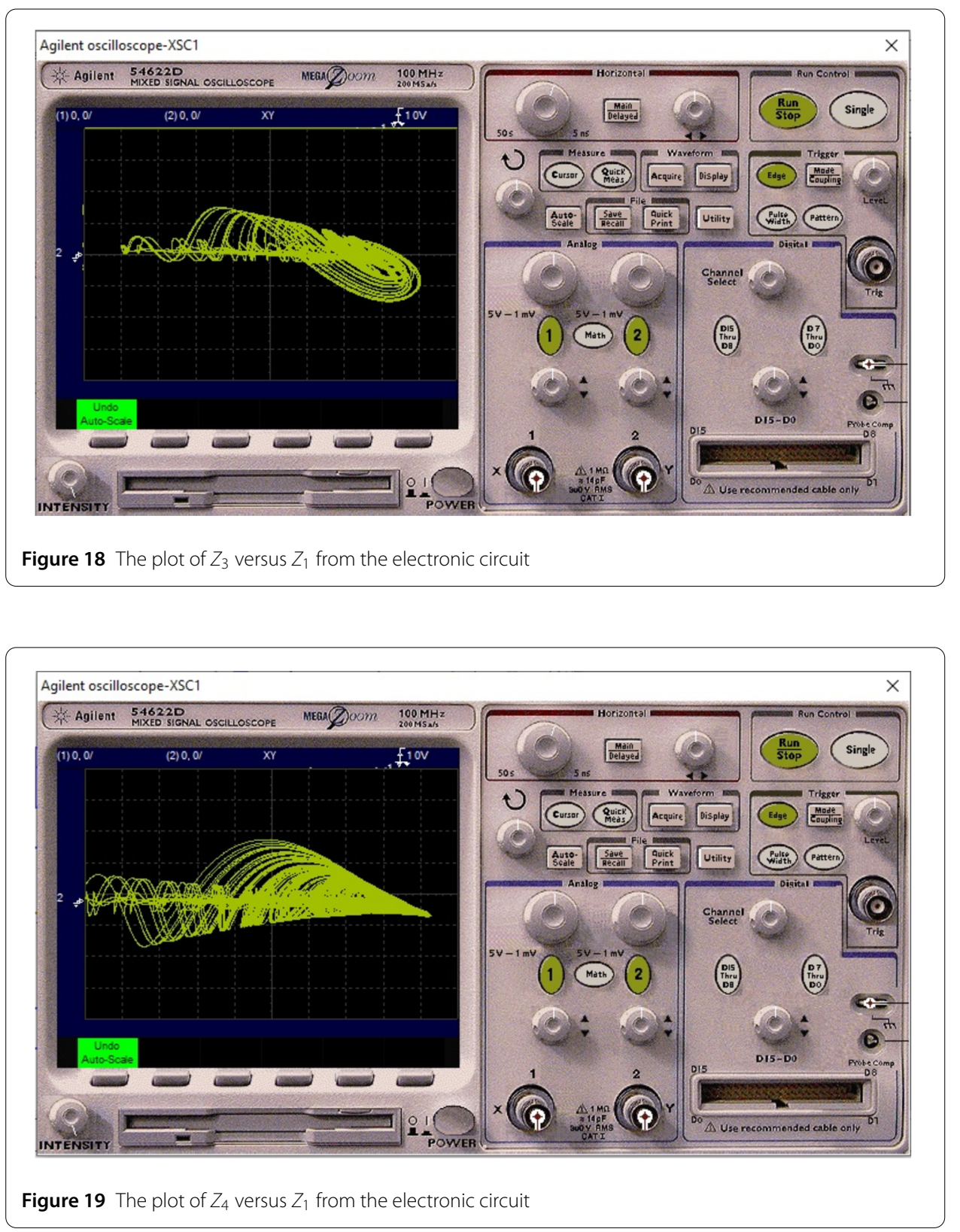

\section{Conclusion}

In this paper, the fractional snap system with only one nonlinear term has been analyzed using various tools such as L.E., equilibrium point analysis, bifurcation diagram, and dissipativity. The system is synchronized in triple compound combination antisynchronization considering uncertainties and disturbances among parallel systems using two control methods. Comparison of the obtained results with previous synchronization literature is made. The achieved synchronization has been illustrated in security of information transmission. Also the circuit of the system has been implemented.

The future scope of this work lies in studying the hidden attractors of the introduced fractional snap system. The achieved synchronization can be made applicable in the field of image and voice encryption as well. 
Acknowledgements

Taif University Researchers Supporting Project number (TURSP-2020/20), Taif University, Taif, Saudi Arabia.

\section{Funding}

This paper is funded by Taif University Researchers Supporting Project number (TURSP-2020/20), Taif University, Taif, Saudi Arabia.

\section{Availability of data and materials}

Not applicable.

\section{Competing interests}

The authors declare that they have no competing interests.

\section{Authors' contributions}

All authors contributed equally to the writing of this paper. All authors read and approved the final manuscript.

\section{Author details}

'Department of Mathematics and Statistics, College of Science, Taif University, P.O. Box 11099, Taif 21944, Saudi Arabia. 2 Department of Mathematics, Faculty of Science, Sohag University, Sohag 82524, Egypt. ${ }^{3}$ Department of Mathematics, Jamia Millia Islamia, New Delhi 110025, India. ${ }^{4}$ Department of Physics and Engineering Mathematics, Faculty of Electronic Engineering, Menoufia University, Menouf 32952, Egypt. ${ }^{5}$ Department of Computer Science and Artificial Intelligence, College of Computer Science and Engineering, University of Jeddah, Jeddah, Saudi Arabia.

\section{Publisher's Note}

Springer Nature remains neutral with regard to jurisdictional claims in published maps and institutional affiliations.

Received: 21 August 2020 Accepted: 31 March 2021 Published online: 20 April 2021

\section{References}

1. Baleanu, D., Ghanbari, B., Asad, J.H., Jajarmi, A., Pirouz, H.M.: Planar system-masses in an equilateral triangle: numerical study within fractional calculus. Comput. Model. Eng. Sci. 124(3), 953-968 (2020)

2. Baleanu, D., Jajarmi, A., Sajjadi, S.S., Asad, J.H.: The fractional features of a harmonic oscillator with position-dependent mass. Commun. Theor. Phys. 72(5), 055002 (2020)

3. Boccaletti, S., Valladares, D.L.: Characterization of intermittent lag synchronization. Phys. Rev. E 62(5), 7497-7500 (2000)

4. Broucke, M.: One parameter bifurcation diagram for Chua's circuit. IEEE Trans. Circuits Syst. 34(2), 208-209 (1987)

5. Buscarino, A., Fortuna, L., Frasca, M., Sciuto, G.: A Concise Guide to Chaotic Electronic Circuits. Springer, Berlin (2014)

6. Chang, D., Li, Z., Wang, M., Zeng, Y.: A novel digital programmable multi-scroll chaotic system and its application in FPGA-based audio secure communication. AEÜ, Int. J. Electron. Commun. 88, 20-29 (2018)

7. Cvetkovi, D., Doob, M., Sachs, H.: Spectra of Graphs Theory and Application, 3rd edn. Verlag, Heidelberg (1995)

8. de la Peña, J.A., Gutman, I., Rada, J.: Estimating the estrada index. Linear Algebra Appl. 427, 70-76 (2007)

9. Diethelm, K., Ford, N.J.: Analysis of fractional differential equations. J. Math. Anal. Appl. 265, 229-248 (2002). https://doi.org/10.1006/jmaa.2000.7194

10. Dongmo, E.D., Ojo, K.S., Woafo, P., Njah, A.N.: Difference synchronization of identical \& non-identical chaotic \& hyper chaotic systems of different orders using active backstepping design. J. Comput. Nonlinear Dyn. 13(5) (2018)

11. Fatemi-Behbahani, E., Ansari-Asl, K., Farshidi, E.: A new approach to analysis and design of chaos-based random number generators using algorithmic converter. Circuits Syst. Signal Process. 35(11), 3830-3846 (2016)

12. Feki, M.: An adaptive chaos synchronization scheme applied to secure communication. Chaos Solitons Fractals 18(1), 141-148 (2003)

13. Geist, K., Parlitz, U., Lauterborn, W.: Comparison of different methods for computing Lyapunov exponents. Prog. Theor. Phys. 83(5), 875-893 (1990)

14. Ibraheem, A.: Dual compound compound synchronization of twelve n-dimensional dynamical systems. Arab. J. Sci. Eng. 1(14) (2020)

15. Jajarmi, A., Baleanu, D.: On the fractional optimal control problems with a general derivative operator. Asian J. Control (2019)

16. Jajarmi, A., Baleanu, D.: A new iterative method for the numerical solution of high-order non-linear fractional boundary value problems. Front. Phys. 8(220) (2020)

17. Jimenez-Triana, A., Chen, G., Gauthier, A.: A parameter-perturbation method for chaos control to stabilizing UPOs. IEEE Trans. Circuits Syst. II, Express Briefs 62(4), 407-411 (2015)

18. Karimi, S., Effati, S., Ghane, F.H.: The synchronization of chaotic systems applying the parallel synchronization method. Phys. Scr. 94(10), 105215 (2019)

19. Khan, A., Trikha, P.: Compound difference anti-synchronization between chaotic systems of integer and fractional order. SN Appl. Sci. 1(7), 757 (2019)

20. Khan, A., Trikha, P.: Study of Earth's changing polarity using compound difference synchronization. GEM Int. J. Geomath. 11(1), 7 (2020)

21. Khan, A., Trikha, P., Jahanzaib, L.S.: Secure communication: using synchronization on a novel fractional order chaotic system. In: 2019 International Conference on Power Electronics, Control and Automation (ICPECA), pp. 1-5. IEEE Comput. Soc., Los Alamitos (2019)

22. Khan, A., Trikha, P., Jahanzaib, L.S.: Dislocated hybrid synchronization via tracking control \& parameter estimation methods with application. Int. J. Model. Simul. 1(11) (2020) 
23. Khan, M.F., Baig, F., Beg, S.: Steganography between silence intervals of audio in video content using chaotic maps. Circuits Syst. Signal Process. 32(11), 3901-3919 (2014)

24. Li, B., Zhou, X., Wang, Y.: Combination synchronization of three different fractional-order delayed chaotic systems. Complexity 2019, Article ID 5184032 (2019)

25. Li, C., Sprott, J.C., Thio, W.: Linearization of the Lorenz system. Phys. Lett. A 379(10-11), 888-893 (2015)

26. Lu, J., Chen, G., Cheng, D., Celikovsky, S.: Bridge the gap between the Lorenz system and the Chen system. Int. J. Bifurc. Chaos 12(12), 2917-2926 (2002)

27. Luo, A.C.J.: A theory for synchronization of dynamical systems. Commun. Nonlinear Sci. Numer. Simul. 14(5), 1901-1951 (2009)

28. Mahmoud, E.E., Higazy, M., Al-Harth, T.M.: Signal flow graph and control of realizable autonomous nonlinear Chen model with quaternion variables. Alex. Eng. J. (2020). https://doi.org/10.1016/j.aej.2020.02.021

29. Mahmoud, G.M., Abed-Elhameed, T.M., Farghaly, A.A.: Double compound combination synchronization among eight n-dimensional chaotic systems. Chin. Phys. B 27, 8 (2018)

30. Mahmoud, G.M., Mahmoud, E.E.: Phase and antiphase synchronization of two identical hyperchaotic complex nonlinear systems. Nonlinear Dyn. 61(1-2), 141-152 (2010)

31. Mahmoud, G.M., Mahmoud, E.E.: Lag synchronization of hyperchaotic complex nonlinear systems. Nonlinear Dyn. 67(2), 1613-1622 (2012)

32. Mainieri, R., Rehacek, J.: Projective synchronization in three-dimensional chaotic systems. Phys. Rev. Lett. 82(15), 3042 (1999)

33. Matignon, D.: Stability results for fractional differential equations with applications to control processing. In: Computational Engineering in Systems Applications, vol. 2, pp. 963-968. IEEE Comput. Soc., Los Alamitos (1996)

34. Mohammadi, F., Moradi, L., Baleanu, D., Jajarmi, A.: A hybrid functions numerical scheme for fractional optimal control problems: application to nonanalytic dynamic systems. J. Vib. Control 24(21), 5030-5043 (2018)

35. Park, J.H.: Adaptive synchronization of hyperchaotic Chen system with uncertain parameters. Chaos Solitons Fractals 26(3), 959-964 (2005)

36. Pecora, L.M., Carroll, T.L.: Synchronization in chaotic systems. Phys. Rev. Lett. 64(8), 821 (1990)

37. Pham, V.T., Jafari, S., Wang, X., Ma, J.: A chaotic system with different shapes of equilibria. Int. J. Bifurc. Chaos 26(04), 1650069 (2016)

38. Pham, V.T., Vaidyanathan, S., Volos, C., Jafari, S., Alsaadi, F.E., Alsaadi, F.E.: Chaos in a simple snap system with only one nonlinearity, its adaptive control and real circuit design. Arch. Control Sci. 29, 73-96 (2019)

39. Prajapati, N., Khan, A., Khattar, D.: On multi switching compound synchronization of non identical chaotic systems. Chin. J. Phys. 56(4), 1656-1666 (2018)

40. Rosenblum, M.G., Pikovsky, A.S., Kurths, J.: Phase synchronization of chaotic oscillators. Phys. Rev. Lett. 76(11), 1804-1807 (1996)

41. Rosenblum, M.G., Pikovsky, A.S., Kurths, J.: From phase to lag synchronization in coupled chaotic oscillators. Phys. Rev. E 78(22), 4193 (1997)

42. Rulkov, N.F., Sushchik, M.M., Tsimring, L.S., Abarbanel, H.D.I.: Generalized synchronization of chaos in directionally coupled chaotic systems. Phys. Rev. E 51(2), 980-994 (1995)

43. Runzi, L., Yinglan, W., Shucheng, D.: Combination synchronization of three classic chaotic systems using active backstepping design. Chaos, Interdiscip. J. Nonlinear Sci. 21(4), 043114 (2011)

44. Sajjadi, S.S., Baleanu, D., Jajarmi, A., Pirouz, H.M.: A new adaptive synchronization and hyperchaos control of a biological snap oscillator. Chaos Solitons Fractals 138, 109919 (2020)

45. Scapellato, R., El-Shanawany, R., Higazy, M.: Orthogonal double covers of Cayley graphs. Discrete Appl. Math. 157(14), 3111-3118 (2009). https://doi.org/10.1016/j.dam.2009.06.005

46. Seyedzadeh, S.M., Mirzakuchaki, S.: A fast color image encryption algorithm based on coupled two-dimensional piecewise chaotic map. Signal Process. 92(5), 1202-1215 (2012)

47. Singh, A.K., Yadav, V.K., Das, S.: Comparative study of synchronization methods of fractional order chaotic systems. Nonlinear Eng. 5(3), 185-192 (2016)

48. Skardal, P.S., Sevilla-Escoboza, R., Vera-Avila, V., Buldu, J.M.: Optimal phase synchronization in networks of phase-coherent chaotic oscillators. Chaos, Interdiscip. J. Nonlinear Sci. 27(1), 013111 (2017)

49. Sun, J., Li, N., Wang, Y., Wang, W.: A novel chaotic system and its modified compound synchronization. Fundam. Inform. 164(2-3), 259-275 (2019)

50. Sun, J., Shen, Y., Yin, Q., Yu, C.: Compound synchronization of four memristor chaotic oscillator systems and secure communication. Chaos, Interdiscip. J. Nonlinear Sci. 23(1), 013140 (2013)

51. Sun, J., Yin, Q., Shen, Y.: Compound synchronization for four chaotic systems of integer order and fractional order. Europhys. Lett. 106(4), 40005 (2014)

52. Tavazoei, M.S., Haeri, M.: A necessary condition for double scroll attractor existence in fractional-order systems. Phys. Lett. A 367, 102-113 (2007)

53. Torrieri, D.J.: Principles of Secure Communication Systems. Artech House, Norwood (1985)

54. Trikha, P., Jahanzaib, L.S.: Secure communication: using double compound-combination hybrid synchronization. In: Proceedings of International Conference on Artificial Intelligence and Applications, pp. 81-91. Springer, Berlin (2020)

55. Vaidyanathan, S.: Generalised projective synchronisation of novel 3-d chaotic systems with an exponential non-linearity via active and adaptive control. Int. J. Model. Identif. Control 22(3), 207-217 (2014)

56. Vaidyanathan, S., Volos, C.K., Kyprianidis, I., Stouboulos, I., Pham, V.T.: Analysis, adaptive control and anti-synchronization of a six-term novel jerk chaotic system with two exponential nonlinearities and its circuit simulation. J. Eng. Sci. Technol. Rev. 8(2), 24-36 (2015)

57. Vidyasagar, M.: Nonlinear Systems Analysis, vol. 42. SIAM, Philadelphia (2002)

58. Wang, X., Teng, L., Qin, X.: A novel colour image encryption algorithm based on chaos. Signal Process. 92(4), $1101-1108(2012)$

59. Wolf, A., Swift, J.B., Swinney, H.L., Vastano, J.A.: Determining Lyapunov exponents from a time series. Phys. D, Nonlinear Phenom. 16(3), 285-317 (1985)

60. Wong, K., Man, K.P., Li, S., Liao, X.: A more secure chaotic cryptographic scheme based on the dynamic look-up table. Circuits Syst. Signal Process. 24(5), 571-584 (2005) 
61. Wu, G.C., Baleanu, D.: Discrete fractional logistic map and its chaos. Nonlinear Dyn. 75(1-2), 283-287 (2014)

62. Wu, G.C., Baleanu, D., Xie, H.P., Chen, F.L.: Chaos synchronization of fractional chaotic maps based on the stability condition. Phys. A, Stat. Mech. Appl. 460, 374-383 (2016)

63. Wu, G.C., Deng, Z.G., Baleanu, D., Zeng, D.Q.: New variable-order fractional chaotic systems for fast image encryption. Chaos, Interdiscip. J. Nonlinear Sci. 29(8), 083103 (2019)

64. Wu, G.C., Luo, M., Huang, L.L., Banerjee, S.: Short memory fractional differential equations for new memristor and neural network design. Nonlinear Dyn. 100, 3611-3623 (2020)

65. Wu, Y., Noonan, J.P., Yang, G., Jin, H.: Image encryption using the two-dimensional logistic chaotic map. J. Electron. Imaging 21(1), 013014 (2012)

66. Yadav, V.K., Prasad, G., Srivastava, M., Das, S.: Triple compound synchronization among eight chaotic systems with external disturbances via nonlinear approach. Differ. Equ. Dyn. Syst., 1-24 (2019)

67. Zhang, B., Deng, F.: Double-compound synchronization of six memristor-based Lorenz systems. Nonlinear Dyn. 77(4), 1519-1530 (2014)

68. Zhang, H., Liu, D., Wang, Z.: Controlling Chaos: Suppression, Synchronization and Chaotification. Springer, Berlin (2009)

\section{Submit your manuscript to a SpringerOpen ${ }^{\circ}$} journal and benefit from:

- Convenient online submission

$\checkmark$ Rigorous peer review

- Open access: articles freely available online

- High visibility within the field

- Retaining the copyright to your article

Submit your next manuscript at $\gg$ springeropen.com 